\title{
WHITNEY LEVELS IN HYPERSPACES OF CERTAIN PEANO CONTINUA
}

\author{
BY
}

JACK T. GOODYKOONTZ, JR. AND SAM B. NADLER, JR.

\begin{abstract}
Let $X$ be a Peano continuum. Let $2^{X}$ (resp., $C(X)$ ) be the space of all nonempty compacta (resp., subcontinua) of $X$ with the Hausdorff matric. Let $\omega$ be a Whitney map defined on $\mathcal{K}=2^{X}$ or $C(X)$ such that $\omega$ is admissible (this requires the existence of a certain type of deformation of $\mathcal{H})$. If $\mathcal{H}=C(X)$, assume $X$ contains no free arc. Then, for any $t_{0} \in(0, \omega(X))$, it is proved that $\omega^{-1}\left(t_{0}\right), \omega^{-1}\left(\left[0, t_{0}\right]\right)$, and $\omega^{-1}\left(\left[t_{0}, \omega(X)\right]\right)$ are Hilbert cubes. This is an analogue of the Curtis-Schori theorem for $\mathcal{H}$. A general result for the existance of admissible Whitney maps is proved which implies that these maps exist when $X$ is starshaped in a Banach space or when $X$ is a dendrite. Using these results it is shown, for example that being an AR, an ANR, an LC space, or an $\mathrm{LC}^{n}$ space is not strongly Whitney-reversible.
\end{abstract}

Introduction. A continuum is a nonempty compact connected metric space. A Peano continuum is a locally connected continuum. The hyperspaces of a continuum $X$ are the spaces $2^{X}=\{A \subset X: A$ is nonempty and compact $\}$ and $C(X)=\left\{A \in 2^{X}\right.$ : $A$ is connected $\}$ metrized with the Hausdorff metric [21]. Let $F_{1}(X)=\{\{x\}$ : $x \in X\}$. A Whitney map for a hyperspace $\mathcal{H}$ of $X$ is a continuous function $\omega$ : $\mathcal{H} \rightarrow[0,+\infty)$ such that $\omega(\{x\})=0$ for each $\{x\} \in F_{1}(X)$ and if $A, B \in \mathcal{H}$ and $A \subset B \neq A$, then $\omega(A)<\omega(B)$. Intuitively, a Whitney map is a size function. If $\omega$ is a Whitney map for $\mathcal{H}$ and $0 \leqslant t<\omega(X)$ then $\omega^{-1}(t)$ is called a Whitney level; if $0<t<\omega(X)$, then $\omega^{-1}(t)$ is called a positive Whitney level. Thus, a Whitney level consists of compacta or continua in $X$ of the same "size". Basic facts about Whitney maps and Whitney levels are in [21]. We note that Whitney levels are coverings of $X$ which, as $t$ gets close to zero, converge to $\omega^{-1}(0)$ and consist of compacta of small diameter. Note that $\omega^{-1}(0)=F_{1}(X)$ and, hence, $\omega^{-1}(0)$ is homeomorphic to $X$. Thus, as $t$ gets close to zero, the Whitney levels "approximate" $X$. It is of interest to obtain information about the structure of positive Whitney levels and to determine those properties which are preserved by the convergence of positive Whitney levels to the zero level. Resultsa about this could be used to determine properties of $X$. This is in analogy with the use of nerves of covers - the nerves correspond to polyhedra, these polyhedra "approximate" the original space as the mesh of the covers approaches zero, and properties of these polyhedra determine various properties of the original space.

Received by the editors October 19, 1981.

1980 Mathematics Subject Classification. Primary 54B20; Secondary 54C99, 54F20.

Key words and phrases. Absolute retract, acyclicity, admissible Whitney map, arc-smooth, cell-like map, contractible, dendrite, dendroid, Hilbert cube, hyperspace, locally $n$-connected, Peano continuum, shape, smooth dendroid, starshaped, strong Whitney-reversible property, Whitney level, Whitney map, Whitney stable, Z-set. 
In this paper our study of the structure of positive Whitney levels focuses on conditions under which positive Whitney levels in hyperspaces of Peano continua are Hilbert cubes. In [7] Curtis and Schori proved that for any Peano continuum $X, 2^{X}$ is a Hilbert cube and, if $X$ contains no free arc, $C(X)$ is a Hilbert cube. It can be shown that if all positive Whitney levels in $2^{X}$ or $C(X)$ are absolute retracts, then $X$ has trivial shape (use ideas in the proof of (2.11)). In 14.61 of [21] it was shown that there is a Whitney map for $2^{X}$ when $X$ is an arc such that some positive Whitney level is not connected (Whitney levels in $C(X)$ are always connected [11, p. 1032]). In [24, pp. 277-278] it was shown that there is a Whitney map for $C(X)$ when $X$ is a 2-cell such that some positive Whitney level is not acyclic. Thus, even when $X$ is an absolute retract, it is not possible to obtain an exact analogue for positive Whitney levels of the Curtis-Schori theorem. However, by imposing a certain general condition on the Whitney map, we do obtain an analogue of the Curtis-Schori theorem. This condition requires that there exist a deformation of the hyperspace which continuously pulls any set of positive Whitney size down to a set of size zero (see (2.1)). If such a deformation exists for a given Whitney map, then we say that the Whitney map is admissible. Our analogue of the Curtis-Schori theorem is in (4.1), and some special cases of (4.1) are in (4.2), (4.6), and (4.8). A similar type of result for "bottoms" and "tops" of hyperspaces is in (4.10). In connection with these results we mention that positive Whitney levels in $2^{X}$ have not been determined before for any continuum $X$. We also note that these results can be used to determine positive Whitney levels in hyperspaces of nonlocally connected continua (see the discussion following (4.12)).

The result in (4.2) is especially important for our investigation into those properties which are preserved by the convergence of positive Whitney levels to the zero level. A topological property $P$ is said to be strongly Whitney-reversible [21, p. 453] provided that whenever $X$ is a continuum such that all positive Whitney levels in $C(X)$ have property $P$ for some Whitney map for $C(X)$, then $X$ has property $P$. In [21 and 23] a number of properties were shown to be strongly Whitney-reversible, e.g., unicoherence, $\operatorname{dim} \leqslant n$, trivial shape, acyclicity, etc. As a simple consequence of (4.2), we obtain the following result in (4.3): If $P$ is a topological property which the Hilbert cube has but which the cone over some Peano continuum does not have, then $P$ is not a strong Whitney-reversible property. Thus, we are able to show that a number of properties are not strongly Whitney-reversible (see (4.4) and (4.5)).

The results in (4.4) and (4.6) answer questions raised in [21]. These questions are discussed in the paragraph following (4.2) and in (4.7).

The paper is organized in the following way. In $\$ 2$ we obtain results about admissible Whitney maps and their Whitney levels. Existence theorems for these maps are in (2.13) through (2.17). The material in $\$ 2$ will be used in the proofs of our main results. Since many of the results in $\$ 2$ are for arbitrary continua, it seems that these results are of interest independent of their applications in this paper. In $\$ 3$ we prove two $Z$-set lemmas for Whitney levels of admissible Whitney maps ((3.2) and (3.3)). These two lemmas are analogues of 5.4 of [8] and are used in the proof of (4.1). In $\S 4$ we state and prove our main results. Near the end of $\S 4$ we raise some questions related to the work in the paper. 
We will use the following symbols. If $A$ and are sets, then $A-B=\{x \in A$ : $x \notin B\}$. If $A$ is a subset of a space $Y$, then $\bar{A}$ denotes the closure of $A$ in $Y$ and $A^{\circ}$ denotes the interior of $A$ in $Y$. If $f: Y \rightarrow Z$ is a function and $A \subset Y$, then $f \mid A$ denotes the restriction of $f$ to $A$. Unless otherwise stated, the letter $X$ will denote a continuum. The symbol $R^{1}$ denotes the real line.

An $\operatorname{arc} A$ in $X$ is said to be a free $\operatorname{arc}$ in $X$ provided that $A$ without its end points is an open subset of $X$.

We will use Torunczyk's recent characterization of the Hilbert cube stated below. Recall that a closed subset $A$ of a continuum $Y$ with metric $d$ is said to be a $Z$-set in $Y$ provided that for each $\varepsilon>0$ there is a continuous function $f_{\varepsilon}: Y \rightarrow Y-A$ such that $d\left(f_{\varepsilon}(y), y\right)<\varepsilon$ for all $y \in Y[5$, p. 2]. A $Z$-map is a continuous function $f$ : $Y \rightarrow Y$ such that $f(Y)$ is a $Z$-set in $Y$ [25, p. 33]. The following result is a special case of Theorem 1 of $[25$, p. 34].

(1.1) THEOREM [25]. Let $Y$ be a compact metric absolute retract. If the identity map on $Y$ is a uniform limit of Z-maps, then $Y$ is a Hilbert cube.

We will use the following result several times. We mention that necessary and sufficient conditions on a metric $d$ in order that $K_{d}$ (defined below) be continuous have been given in $[\mathbf{2 0}, 3.3]$.

(1.2) Theorem. Let $X$ be a Peano continuum. Then, $X$ has a convex metric $[1,19]$. Let $\mathcal{H}=2^{X}$ or $C(X)$, let $d$ be a convex metric for $X$, and define $K_{d}$ on $[0,+\infty) \times \mathcal{H}$ by letting

$$
K_{d}(t, A)=\{x \in X: d(x, a) \leqslant t \text { for some } a \in A\}
$$

for each $(t, A) \in[0,+\infty) \times \mathcal{H}$. Then, $K_{d}$ maps $[0,+\infty) \times \mathcal{H}$ into $\mathcal{H}$ and $K_{d}$ is continuous.

We assume in this paper that the reader is familiar with basic ideas from hyperspace theory. Of particular importance is the notion and the properties of order arcs-see [21, pp. 56-81].

We will use the notion of shape due to Borsuk. The reader may wish to see [16, 2.1] for a number of conditions each of which is equivalent to trivial shape for a compactum. A cell-like map (or CE map) is a continuous function whose point inverses all have trivial shape.

2. Admissible Whitney maps. The necessity of using special types of Whitney maps in this paper is discussed in $\$ 1$. The notion of an admissible Whitney map is defined in (2.1). In (2.2) we show that the existence of admissible Whitney maps is a topological invariant. In (2.3) through (2.11) we determine some basic properties of $X$ and of positive Whitney levels under the assumption that there is an admissible Whitney map. In order to use (1.1) later, it is crucial to know that these levels are absolute retracts. This is proved in (2.9). In (2.13) we give a sufficient condition for the existence of admissible Whitney maps. The significance of (2.13) is evident from the applications of (2.14) through (2.16) in $\$ 4$ (see (4.2) through (4.8)). 
(2.1) Definitions. Let $\mathcal{H}$ denote either one of the hyperspaces $2^{X}$ or $C(X)$. A Whitney map $\omega$ for $\mathcal{H}$ is called an admissible Whitney map for $\mathcal{H}$ provided that there is a (continuous) homotopy $h: \mathcal{H} \times[0,1] \rightarrow \mathcal{H}$ satisfying the following two conditions:

(i) for all $A \in \mathcal{H}, h(A, 1)=A$ and $h(A, 0) \in F_{1}(X)$;

(ii) if $\omega(h(A, t))>0$ for some $A \in \mathcal{H}$ and $t \in[0,1]$, then $\omega(h(A, s))<\omega(h(A, t))$ whenever $0 \leqslant s<t$.

A homotopy $h: \mathcal{H} \times[0,1] \rightarrow \mathcal{H}$ satisfying (i) and (ii) is called an $\omega$-admissible deformation for $\mathcal{H}$. We remark that it is not required in (i) that $h(A, 0)$ be the same singleton for all $A \in \mathcal{H}$ (see(2.4), (2.5), and (4.14)).

(2.2) Proposition. Let $X$ and $X^{\prime}$ be homeomorphic continua. If there is an admissible Whitney map $\omega$ for $2^{X}$ (respectively, $C(X)$ ), then there is an admissible Whitney map $\omega^{\prime}$ for $2^{X^{\prime}}$ (respectively, $C\left(X^{\prime}\right)$ ).

Proof. Assume that $\omega$ is an admissible Whitney map for $2^{X}$, and let $h$ be an $\omega$-admissible deformation for $2^{X}$. Let $f$ be a homeomorphism from $X^{\prime}$ onto $X$. Define $\omega^{\prime}$ on $2^{X^{\prime}}$ by letting $\omega^{\prime}\left(A^{\prime}\right)=\omega\left(f\left[A^{\prime}\right]\right)$ for each $A^{\prime} \in 2^{X^{\prime}}$. Note that, since $f$ is a homeomorphism, $\omega^{\prime}$ is a Whitney map for $2^{X^{\prime}}$. Define $h^{\prime}: 2^{X^{\prime}} \times[0,1] \rightarrow 2^{X^{\prime}}$ by letting

$$
h^{\prime}\left(A^{\prime}, t\right)=f^{-1}\left(h\left(f\left[A^{\prime}\right], t\right)\right)
$$

for all $\left(A^{\prime}, t\right) \in 2^{X^{\prime}} \times[0,1]$. It follows easily that $h^{\prime}$ is an $\omega^{\prime}$-admissible deformation for $2^{X^{\prime}}$. Therefore, $\omega^{\prime}$ is an admissible Whitney map for $2^{X^{\prime}}$. The proof for the case of $C(X)$ and $C\left(X^{\prime}\right)$ is done the same way.

The following four results give facts about $X$ when there is an admissible Whitney map for $2^{X}$ or $C(X)$. Another such result is in (2.11).

(2.3) THEOREM. If there is an admissible Whitney map $\omega$ for $\mathcal{H}=2^{X}$ or $C(X)$, then $X$ is arcwise connected.

Proof. Let $h$ be an $\omega$-admissible deformation for $H$. By (i) of $(2.1), h(\mathcal{H}, 0) \subset$ $F_{1}(X)$. Thus, since $\mathcal{H}$ is arcwise connected [21, 1.13], $h(\mathcal{H}, 0)$ is an arcwise connected subset of $F_{1}(X)$. Fix a point $\{x\} \in F_{1}(X)$. Let $\mathcal{Q}=\{h(\{x\}, t): t \in[0,1]\}$. By (i) of (2.1), $h(\{x\}, 1)=\{x\}$. Hence, $\omega(h\{x\}, 1)=0$. Thus, it follows from (ii) of (2.1) that $A \subset F_{1}(X)$. Hence, since $Q$ is arcwise connected, there is an arc in $F_{1}(X)$ from $h(\{x\}, 1)=\{x\}$ to $h(\{x\}, 0)$. Thus, we have proved that each point $\{x\} \in F_{1}(X)$ can be joined by an arc in $F_{1}(X)$ to a point in $h(\mathcal{H}, 0)$. Therefore, since $h(\mathcal{H}, 0)$ is an arcwise connected subset of $F_{1}(X)$, it follows that $F_{1}(X)$, hence $X$, is arcwise connected. This completes the proof of (2.3).

An important application of the following general result is in (2.5). A sharper form of the result is in (2.8).

(2.4) Theorem. Assume that there is an admissible Whitney map w for $\mathcal{H}=2^{X}$ or $C(X)$. Then, $X$ is contractible if and only if $\mathcal{H}$ is contractible. 
Proof. Assume that $\mathcal{H}$ is contractible. It follows from the proof of 3.1 of [13] that there is a contradiction $G: \mathcal{H} \times[0,1] \rightarrow \mathcal{H}$ such that for all $A \in \mathcal{H}, G(A, 0)=$ $A, G(A, 1)=X$, and, if $0 \leqslant t^{\prime} \leqslant t^{\prime \prime} \leqslant 1, G\left(A, t^{\prime}\right) \subset G\left(A, t^{\prime \prime}\right)$. Let $h$ be an $\omega$-admissible deformation for $\mathcal{H}$. Define $f$ on $F_{1}(X) \times[0,1]$ by

$$
f(\{x\}, t)= \begin{cases}h(\{x\}, 1-2 t), & 0 \leqslant t \leqslant \frac{1}{2} \\ h(G(\{x\}, 2 t-1), 0), & \frac{1}{2} \leqslant t \leqslant 1\end{cases}
$$

It follows from (2.1) that $f$ maps $F_{1}(X) \times[0,1]$ into $F_{1}(X)$. Also, for each $\{x\} \in$ $F_{1}(X), f(\{x\}, 0)=\{x\}$ and $f(\{x\}, 1)=h(X, 0)$. Hence, $F_{1}(X)$, therefore $X$, is contractible. This proves half of (2.4). It follows immediately from 3.1 of [13] that if $X$ is contractible, then $\mathcal{H}$ is contractible (no assumption that there is an admissible Whitney map is necessary). Therefore, we have proved (2.4).

(2.5) COROLlaRY. If there is an admissible Whitney map for $2^{X}$ or $C(X)$ and if $X$ is a Peano continuum, then $X$ is contractible.

Proof. If $X$ is a Peano continuum, then $2^{X}$ and $C(X)$ are contractible [30]. Using this fact, (2.5) follows from (2.4).

Under the hypotheses of (2.5), we will see (in (2.9)) that all positive Whitney levels are absolute retracts. However, it follows using (2.15) that $X$ in (2.5) need not be an absolute retract. Let us note the following consequence of (2.5).

(2.6) COROLlary. If there is an admissible Whitney map for a hyperspace of a (compact connected) absolute neighborhood retract $X$, the $X$ is an absolute retract.

Proof. Use (2.5) and [2, 9.1, p. 96].

(2.7) THEOREM. If $\omega$ is an admissible Whitney map for $\mathcal{H}=2^{X}$ or $C(X)$, then, for any $t_{0}$ such that $0<t_{0}<\omega(X), \omega^{-1}\left(t_{0}\right)$ is a retract of $\omega^{-1}\left(\left[t_{0}, \omega(X)\right]\right)$.

Proof. Let $h: \mathcal{H} \times[0,1] \rightarrow \mathcal{K}$ be an $\omega$-admissible deformation for $\mathcal{H}$. Fix $t_{0}$ such that $0<t_{0}<\omega(X)$, and let $\Gamma=\omega^{-1}\left(\left[t_{0}, \omega(X)\right]\right)$. Let $A \in \Gamma$. Since $h(A, 1)=$ $A, \omega(h(A, 1)) \geqslant t_{0}$ and, since $h(A, 0) \in F_{1}(X), \omega(h(A, 0))=0$. Thus, by the continuity of $h$ and $\omega$, there exists $t_{A} \in[0,1]$ such that $\omega\left(h\left(A, t_{A}\right)\right)=t_{0}$; furthermore, since $\omega\left(h\left(A, t_{A}\right)\right)>0$, we have by (ii) of (2.1) that there is only one such $t_{A}$. Hence, we can define a function $\theta: \Gamma \rightarrow[0,1]$ by letting, for each $A \in \Gamma, \theta(A)$ be the unique number in $[0,1]$ such that $\omega(h(A, \theta(A)))=t_{0}$. Using the compactness of $[0,1]$ and the continuity of $h$ and $\omega$, an easy sequence argument shows that the function $\theta$ is continuous. Define $r: \Gamma \rightarrow \omega^{-1}\left(t_{0}\right)$ by letting $r(A)=h(A, \theta(A))$ for each $A \in \Gamma$. Since $\theta$ and $h$ are continuous, $r$ is continuous. If $A \in \omega^{-1}\left(t_{0}\right)$, then $\theta(A)=1$ and, thus, $r(A)=A$. Therefore, $r$ is a retraction from $\Gamma$ onto $\omega^{-1}\left(t_{0}\right)$. This completes the proof of (2.7).

By using (2.7) we obtain the following more definitive version of half of (2.4).

(2.8) Theorem. Assume that there is an admissible Whitney map $\omega$ for $\mathcal{H}=2^{X}$ or $C(X)$. If $\mathcal{H}$ is contractible, then $\omega^{-1}\left(t_{0}\right)$ is contractible for each $t_{0} \in[0, \omega(X)]$. 
Proof. Fix $t_{0} \in[0, \omega(X)]$. If $t_{0}=0$, then $\omega^{-1}\left(t_{0}\right)$ is homeomorphic to $X$ and, hence, by (2.4), $\omega^{-1}\left(t_{0}\right)$ is contractible. Thus, we assume that $t_{0}>0$. We also assume that $t_{0}<\omega(X)$ since if $t_{0}=\omega(X), \omega^{-1}\left(t_{0}\right)=\{X\}$ and, hence, is contractible. Let $G$ : $\mathcal{H} \times[0,1] \rightarrow \mathcal{H}$ be as in the proof of (2.4). Let $\Gamma=\omega^{-1}\left(\left[t_{0}, \omega(X)\right]\right)$. It follows from the properties of $G$ that $G \mid \Gamma \times[0,1]$ maps $\Gamma \times[0,1]$ into $\Gamma$. Hence, $\Gamma$ is contractible. By $(2.7), \omega^{-1}\left(t_{0}\right)$ is a retract of $\Gamma$. Thus, $\omega^{-1}\left(t_{0}\right)$ is contractible. This completes the proof of (2.8).

By using (2.7) we obtain the following result for Peano continua (compare with (2.5) and (2.6)). This result is important for the proof of (4.1).

(2.9) THEOREM. If there is an admissible Whitney map $\omega$ for $2^{X}$ or $C(X)$ and if $X$ is a Peano continuum, then $\omega^{-1}\left(t_{0}\right)$ is an absolute retract for each $t_{0}$ such that $0<t_{0}<$ $\omega(X)$.

Proof. Since $X$ is a Peano continuum, we have by the remark in [13, p. 29] that $\omega^{-1}\left(\left[t_{0}, \omega(X)\right]\right)$ is an absolute retract. Therefore, by $(2.7), \omega^{-1}\left(t_{0}\right)$ is an absolute retract.

The following general result determines many properties of positive Whitney levels when the Whitney map is admissible.

(2.10) TheOREM. If $\omega$ is an admissible Whitney map for $\mathcal{H}=2^{X}$ or $C(X)$, then, for each $t_{0}$ such that $0<t_{0}<\omega(X), \omega^{-1}\left(t_{0}\right)$ has all those properties which are common to all r-images of all hyperspaces. In particular, $\omega^{-1}\left(t_{0}\right)$ is an arcwise connected continuum which has trivial shape (and, thus, is acyclic).

Proof. Fix $t_{0}$ such that $0<t_{0}<\omega(X)$, and let $\Gamma=\omega^{-1}\left(\left[t_{0}, \omega(X)\right]\right)$. We see that $\Gamma$ is a continuum by using [13,2.3] with $A_{1}=X$ if $\mathcal{H}=2^{X}$ and, in addition, using $[13,2.6]$ if $\mathcal{H}=C(X)$. Hence, $C(\Gamma)$ is a hyperspace (of a continuum). For each $\Theta \in C(\Gamma)$, let $f(\Theta)=\cup Q$. By [13, p. 23], $f$ is a continuous function from $C(\Gamma)$ into $2^{X}$. If $\mathcal{H}=C(X)$, then each $\mathbb{Q} \in C(\Gamma)$ is a subcontinuum of $C(X)$ and, thus, by [13,1.2], $f$ maps $C(\Gamma)$ into $C(X)$. Hence, whether $\mathcal{H}=2^{X}$ or $C(X), f$ is a continuous function from $C(\Gamma)$ into $\mathcal{H}$. Therefore, since $f(\mathbb{Q}) \supset A$ for any $A \in \mathbb{Q} \in C(\Gamma), f$ maps $C(\Gamma)$ into $\Gamma$. Let $g: \Gamma \rightarrow C(\Gamma)$ be given by $g(A)=\{A\}$ for each $A \in \Gamma$. Then, $g$ is a right inverse of $f$. Therefore, $f$ is an $r$-map from $C(\Gamma)$ onto $\Gamma$. By (2.7), there is a retraction $r$ from $\Gamma$ onto $\omega^{-1}\left(t_{0}\right)$. Hence, $r \circ f$ is an $r$-map from $C(\Gamma)$ onto $\omega^{-1}\left(t_{0}\right)$. Thus, we have proved that $\omega^{-1}\left(t_{0}\right)$ is an $r$-image of a hyperspace. This completes the proof of (2.10) since the properties listed in the second part of (2.10) are known to be $r$-invariants and are known to be properties of all hyperspaces (see [21]).

By (2.10), all positive Whitney levels have trivial shape when the Whitney map is admissible. We now show that $X$ has trivial shape.

(2.11) THEOREM. If there is an admissible Whitney map $\omega$ for $\mathcal{H}=2^{X}$ or $C(X)$, then $X$ has trivial shape.

Proof. Let $t$ be any number such that $0<t<\omega(X)$. By (2.7), there is a retraction $r_{t}$ from $\omega^{-1}([t, \omega(X)])$ onto $\omega^{-1}(t)$. Define $\bar{r}_{t}$ by

$$
\bar{r}_{t}(A)= \begin{cases}r_{t}(A), & \text { if } A \in \omega^{-1}([t, \omega(X)]), \\ A, & \text { if } A \in \omega^{-1}([0, t]) .\end{cases}
$$


We see that $\bar{r}_{t}$ is a retraction from $\mathcal{H}$ onto $\omega^{-1}([0, t])$. Thus, since $\mathcal{H}$ has trivial shape [21, p. 180], it follows easily that $\omega^{-1}([0, t])$ has trivial shape (use $\left.[16,2.1]\right)$. Observe that

$$
F_{1}(X)=\cap\left\{\omega^{-1}([0, t]): 0<t<\omega(X)\right\} .
$$

Hence, $F_{1}(X)$ is a nested intersection of compacta having trivial shape. Thus, it follows easily that $F_{1}(X)$ has trivial shape (use $[16,2.1]$ ). Therefore, since $F_{1}(X)$ is homeomorphic to $X, X$ has trivial shape.

Whitney maps for $C(X)$ are always monotone and open [11, p. 1032], but Whitney maps for $2^{X}$ may be neither monotone nor open even when $X$ is an are [21, 14.61]. The following result shows that admissible Whitney maps for $C(X)$ or $2^{X}$ are cell-like and open. When this result is combined with (2.13), we obtain a stronger and more general result than 14.66 of [21]. The notion of a cell-like map is defined at the end of $\S 1$.

(2.12) THEOREM. If $\omega$ is an admissible Whitney map for $\mathcal{H}=2^{X}$ or $C(X)$, then $\omega$ is an open $C E$ map (open means $\omega$ is open as a mapping from $\mathcal{H}$ onto $[0, \omega(X)]$ ).

Proof. Recall that $\omega^{-1}(0)$ is homeormorphic to $X$ and that $\omega^{-1}(\omega(X))$ is the one-point set $\{X\}$. Thus, by (2.10) and (2.11), $\omega$ is a CE map. We now show that $\omega$ is open at each $A \in \mathcal{H}$. Since any Whitney map for $C(X)$ is an open map [11, p. 1032], we assume that $\mathcal{H}=2^{X}$ and that $A \in 2^{X}-C(X)$. Let $t_{0}=\omega(A)$, and let $\mathscr{Q}$ be an open subset of $2^{X}$ such that $A \in \mathcal{Q}$. Let $h$ be an $\omega$-admissible deformation for $2^{X}$. By the continuity of $h$, there exists $s_{1}<1$ such that $h(A, s) \in \mathcal{Q}$ for all $s \in\left(s_{1}, 1\right]$. Let $\Sigma_{1}=\left\{h(A, s): s_{1}<s \leqslant 1\right\}$. Since $A \notin F_{1}(X)$ and $h(A, 1)=Q, \omega(h(A, 1))>0$. Hence, by (ii) of (2.1), $\omega\left(\Sigma_{1}\right)$ contains a nonempty half-open interval of the form $\left(t_{1}, t_{0}\right]$. Let $\sigma:[0,1] \rightarrow 2^{X}$ be a segment from $A$ to $X[13,2.3]$. By the continuity of $\sigma$, there exists $s_{2}>0$ such that $\sigma(s) \in \mathcal{Q}$ for all $s \in\left[0, s_{2}\right)$. Let $\Sigma_{2}=\left\{\sigma(s): 0 \leqslant s<s_{2}\right\}$. Since $A \neq X, \omega \mid \Sigma_{2}$ is a homeomorphism from $\Sigma_{2}$ onto a (nonempty) half-open interval of the form $\left[t_{0}, t_{2}\right)$. We have that

$$
t_{0} \in\left(t_{1}, t_{2}\right) \subset \omega\left(\Sigma_{1} \cup \Sigma_{2}\right) \subset \omega(\Omega) .
$$

It follows that $\omega$ is open at $A$. This completes the proof of (2.12).

An application of (2.12) is in (4.12).

We have determined various properties of Whitney levels when the Whitney map is admissible. The following result gives a useful sufficient condition for the existence of admissible Whitney maps.

(2.13) Proposition. Let $X$ be a continuum with metric $d$ such that there is a homotpy $\psi: X \times[0,1] \rightarrow X$ satisfying the following two conditions:

(1) $\psi(x, 1)=x$ and, for some given point $p \in X, \psi(x, 0)=p$ for all $x \in X$;

(2) if $d\left(\psi\left(x_{1}, t\right), \psi\left(x_{2}, t\right)\right)>0$ for some $x_{1}, x_{2} \in X$ and $t \in[0,1]$, then

$$
d\left(\psi\left(x_{1}, s\right), \psi\left(x_{2}, s\right)\right)<d\left(\psi\left(x_{1}, t\right), \psi\left(x_{2}, t\right)\right) \text { whenever } 0 \leqslant s<t .
$$

Then, there are admissible Whitney maps for $2^{X}$ and $C(X)$. 
Proof. Let $\mathcal{H}=2^{X}$ or $C(X)$. Define $h: \mathcal{H} \times[0,1] \rightarrow \mathcal{H}$ by letting $h(A, t)=$ $\{\psi(a, t): a \in A\}$ for each $A \in \mathcal{K}$ and each $t \in[0,1]$. Since $\psi$ is uniformly continuous, $h$ is continuous. It follows easily using (1) and the definition of $h$ that for all $A \in \mathcal{H}, h(A, 1)=A$ and $h(A, 0)=\{p\} \in F_{1}(X)$. We will show that $h$ is an $\omega$-admissible deformation for $\mathcal{H}$ when $\omega$ is the Whitney map for $\mathcal{H}$ as defined in [28, p. 275]. We include the definition of $\omega$ here. Let $B \in \mathcal{H}$. For each $n \geqslant 2$ let $F_{n}(B)=$ $\{K \subset B: K \neq \varnothing$ and the cardinality of $K$ is $\leqslant n\}$, define $\lambda_{n}: F_{n}(B) \rightarrow[0,+\infty)$ by letting $\lambda_{n}\left(\left\{b_{1}, \ldots, b_{n}\right\}\right)=\min \left\{d\left(b_{i}, b_{j}\right): i \neq j\right\}$ for all $\left\{b_{1}, \ldots, b_{n}\right\} \in F_{n}(B)$, and let $\omega_{n}(B)=\sup \lambda_{n}\left[F_{n}(B)\right]$. Then,

$$
\omega(B)=\sum_{n=2}^{\infty} 2^{1-n} \cdot \omega_{n}(B) .
$$

Let $A \in \mathcal{H}$ and $t \in[0,1]$ such that $\omega(h(A, t))>0$. Let $s$ be such that $0 \leqslant s<t$. Fix $n \geqslant 2$. Note that $F_{n}(h(A, s))$ is compact and that $\lambda_{n}$ is continuous. Hence, $\omega_{n}(h(A, s))=\lambda_{n}(E)$ for some $E \in F_{n}(h(A, s))$. By the definition of $h, E=$ $\left\{\psi\left(a_{1}, s\right), \ldots, \psi\left(a_{n}, s\right)\right\}$ for some $a_{1}, \ldots, a_{n} \in A$. Let $G=\left\{\psi\left(a_{1}, t\right), \ldots, \psi\left(a_{n}, t\right)\right\}$ and note that $G \in F_{n}(h(A, t))$. By the definition of $\lambda_{n}, \lambda_{n}(E)=d\left(\psi\left(a_{i}, s\right), \psi\left(a_{j}, s\right)\right)$ for some $i \neq j$ and $\lambda_{n}(G)=d\left(\psi\left(a_{k}, t\right), \psi\left(a_{m}, t\right)\right)$ for some $k \neq m$. For the purpose of proving (\#) below, assume that $\omega_{n}(h(A, s))>0$, i.e., $\lambda_{n}(E)>0$. Suppose that $\lambda_{n}(G)=0$. Then, by (2) and the continuity of $\psi, d\left(\psi\left(a_{k}, s\right), \psi\left(a_{m}, s\right)\right)=0$. Hence, since $k \neq m$ and $\psi\left(a_{k}, s\right), \psi\left(a_{m}, s\right) \in E, \lambda_{n}(E)=0$, a contradiction. Therefore, $\lambda_{n}(G)>0$. Thus, by (2),

$$
d\left(\psi\left(a_{k}, s\right), \psi\left(a_{m}, s\right)\right)<d\left(\psi\left(a_{k}, t\right), \psi\left(a_{m}, t\right)\right) .
$$

Hence, since $\lambda_{n}(E) \leqslant d\left(\psi\left(a_{k}, s\right), \psi\left(a_{m}, s\right)\right)$, we have that $\lambda_{n}(E)<\lambda_{n}(G)$. Since $G \in F_{n}(h(A, t)), \lambda_{n}(G) \leqslant \omega_{n}(h(A, t))$. Thus, since $\omega_{n}(h(A, s))=\lambda_{n}(E)$, we have that $\omega_{n}(h(A, s))<\omega_{n}(h(A, t))$. Therefore, we have proved the following fact:

$(\sharp)$ If $\omega_{n}(h(A, s))>0$ for a given $n \geqslant 2$, then $\omega_{n}(h(A, s))<\omega_{n}(h(A, t))$.

Since $\omega(h(A, t))>0$, there exists $n^{\prime} \geqslant 2$ such that $\omega_{n^{\prime}}(h(A, t))>0$. It follows from ( $\sharp)$ that

(a) $\omega_{n^{\prime}}(h(A, s))<\omega_{n^{\prime}}(h(A, t))$.

Since $0 \leqslant \omega_{n}(h(A, t))$ for all $n \geqslant 2$, it also follows using ( $\left.\sharp\right)$ that

(b) $\omega_{n}(h(A, s)) \leqslant \omega_{n}(h(A, t))$ for all $n \geqslant 2$.

By (a), (b), and the definition of $\omega$, we see that $\omega(h(A, s))<\omega(h(A, t))$. This completes the proof that $h$ is an $\omega$-admissible deformation for $\mathcal{H}$, i.e., $\omega$ is an admissible Whitney map for $\mathcal{H}$.

The following corollary to (2.13) can be used to see that there are admissible Whitney maps for the hyperspaces of many contractible polyhedra. It can also be used to show that there are admissible Whitney maps for the hyperspaces of certain types of continua which are not necessarily locally connected (for example, see (2.15)). Recall that a subset $S$ of a Banach space $E$ is starshaped provied that there is a point $p \in S$ such that for any $x \in S$, the convex arc in $E$ from $p$ to $x$ lies in $S$; the point $p$ is then called a seeing point of $S$.

(2.14) COROLlaRY. If $X$ is a compact starshaped subset of a Banach space, then there are admissible Whitney maps for $2^{X}$ and $C(X)$. 
Proof. Assume, without loss of generality (by translating $X$ ), that the zero vector of the Banach space is a seeing point of $X$. Then, for any $t \in[0,1]$ and any $x \in X$, the scalar product $t \cdot x$ is a point of $X$. Hence, letting $\psi(x, t)=t \cdot x$ for each $(x, t) \in X \times[0,1]$, we have that $\psi$ maps $X \times[0,1]$ into $X$. Clearly, $\psi$ satisfies (1) of (2.13) and, letting $d$ denote the metric for $X$ obtained from the norm on the Banach space, we see that $\psi$ also satisfies (2) of (2.13). Therefore, (2.14) follows from (2.13).

The following result will be of significant value in $\$ 4$. It will be used to prove (4.2), and (4.2) will be used to obtain some of our main results about strong Whitney-reversible properties (see (4.3)-(4.5)).

(2.15) THEOREM. If $X$ is the (topological) cone over any nonempty compact metric space $Y$, then there are admissible Whitney maps for $2^{X}$ and $C(X)$.

Proof. Embed $Y$ as a subset $Y^{\prime}$ of a Banach space $E\left[2,8.1\right.$, p. 79]. We consider $Y^{\prime}$ as a subset of the subspace $E \times\{0\}$ of the Banach space $E \times R^{1}$. Let $v=(e, 1) \in E$ $\times R^{1}$ be fixed. Let $X^{\prime}$ denote the subset of $E \times R^{1}$ obtained by joining each point of $Y^{\prime}$ to $v$ by a convex arc in $E \times R^{1}$. Then, $X^{\prime}$ is a compact starshaped subset of $E \times R^{1}$ and, since $Y$ is compact, $X$ and $X^{\prime}$ are homeomorphic. Therefore, (2.15) now follows from (2.14) and (2.2).

As the proof of the following result shows, (2.13) can be applied to continua which can not be embedded in a Banach space so as to be starshaped. The result will be used in the proof of (4.8). Recall [17, p. 300] that a dendrite is a Peano continuum which contains no simple closed curve.

(2.16) THEOREM. If $X$ is any dendrite, then there are admissible Whitney maps for $2^{X}$ and $C(X)$.

Proof. Let $p \in X$ and, for $y, z \in X$, let $y \leqslant_{p} z$ mean $z$ lies on the (unique) are in $X$ from $p$ to $z$. As is well known, $\left(X, \leqslant_{p}\right)$ is a partially ordered space. Thus, by Theorem 1 of [4], $X$ has a metric $d$ which is radially convex with respect to $\leqslant_{p}$. Furthermore, by the proof in [4, pp. $229-230]$, we see that

$(*)$ if $y \leqslant_{p} z$, then $d(p, z)=d(p, y)+d(y, z)$.

Now define a new metric $D$ for $X$ as follows. Let $y, z \in X$. Let $y \wedge z$ denote the last point (with respect to $\leqslant_{p}$ ) where the arc from $p$ to $y$ intersects the arc from $p$ to $z$. Then, let

$$
D(y, z)=d(y, y \wedge z)+d(y \wedge z, z) .
$$

It is easy to see that $D$ is a metric giving the original topology on $X$. Define $\psi$ : $X \times[0,1] \rightarrow X$ by letting $\psi(x, t)$ denote the unique point on the arc from $p$ to $x$ such that

$$
D(p, \psi(x, t))=t \cdot D(p, x) .
$$

It follows easily that $\psi$ is continuous. Also, $\psi$ satisfies (1) of (2.13) and, by using (*) above, we see that $\psi$ satisfies (2) of (2.13). Therefore, by (2.13), there are admissible Whitney maps for $2^{X}$ and $C(X)$.

A dendroid is an arcwise connected hereditarily unicoherent continuum. A dendroid $X$ is smooth provided that for some point $p \in X$, the familiar weak cut point 
partial order $\leqslant_{p}$ with respect to $p[15]$ is closed (as a subset of $X \times X$ ). The following result will be used in the proof of (4.8). Since every dendrite is a smooth dendroid, the result generalizes and strengthens the part of (2.16) concerning $C(X)$ - see (4.9).

(2.17) THEOREM. If $X$ is any smooth dendriod, then every Whitney map for $C(X)$ is admissible.

Proof. Let $X$ be a dendroid which is smooth at a point $p$, let $\leqslant_{p}$ be as above, and let $\omega$ be an Whitney map for $C(X)$. As in the proof of $(2.16)$, the radically convex metric $d$ for $X$ satisfying (*) exists. Each $A \in C(X)$ has a unique zero, $z(A)$, with respect to $\leqslant_{p}$, and the function $z: C(X) \rightarrow X$ is continuous [3,p. 42]. Now, fix an $A \in C(X)$. Let $\leqslant_{z(A)}$ denote the weak cut point partial order on $A$ with respect to $z(A)$. We note that $A$ is a dendroid which is smooth at $z(A)$, and that the restriction of $d$ to $A$ is a metric which is radially convex with respect to $\leqslant_{z(A)}$. Define $\psi_{A}$ : $A \times[0,1] \rightarrow A$ by letting $\psi_{A}(a, t)$ denote the unique point on the arc from $z(A)$ to $a$ such that

$$
d\left(z(A), \psi_{A}(a, t)\right)=t \cdot d(z(A), a) .
$$

It follows easily that $\psi_{A}$ is continuous. Now define $h: C(X) \times[0,1] \rightarrow C(X)$ by letting, for each $(A, t) \in C(X) \times[0,1]$,

$$
h(A, t)=\left\{\psi_{A}(a, t): a \in A\right\} .
$$

The continuity of $h$ follows from the smoothness of $X$, the continuity of $z$, and the continuity, for each $A \in C(X)$, of $\psi_{A}$. It follows easily that $h$ is $\omega$-admissible deformation for $C(X)$. Therefore, we have proved (2.17).

3. Two $Z$-set lemmas. The two lemmas proved in (3.2) and (3.3) are analogues for Whitney levels of 5.4 of [8]. The lemmas will be used in the proof of (4.1).

The following proposition is easy to prove. It will be used in this section and in $\S 4$.

(3.1) Proposition. Let $\omega$ be a Whitney map for $\mathcal{H}=2^{X}$ or $C(X)$. Let $\mathcal{S} \subset \mathcal{H}$ and let $\sigma: \mathcal{S} \rightarrow C(\mathcal{H})$ be a continuous function such that for each $B \in \mathcal{S}, \sigma(B)$ is an order arc in $\mathcal{H}$. Let $t_{0} \in[0, \omega(X)]$ and assume that for each $B \in \mathcal{S}, \sigma(B) \cap \omega^{-1}\left(t_{0}\right) \neq 0$. Then, for each $B \in \mathcal{S}, \sigma(B) \cap \omega^{-1}\left(t_{0}\right)$ consists of exactly one point of $\omega^{-1}\left(t_{0}\right)$, denoted by $\sigma_{t_{0}}(B)$, and the function $\sigma_{t_{0}}: \mathcal{S} \rightarrow \omega^{-1}\left(t_{0}\right)$ is continuous.

(3.2) Lemma. Let $X$ be a Peano continuum and let $A$ be a closed subset of $X$ such that $A$ has nonempty interior in $X$. Assume that $A$ does not contain any free arc in $X$. If $\omega$ is an admissible Whitney map for $H=2^{X}$ or $C(X)$, then, for each $t_{0}$ such that $0<t_{0}<\omega(X),\left\{B \in \omega^{-1}\left(t_{0}\right): B \supset A\right\}$ is a Z-set in $\omega^{-1}\left(t_{0}\right)$.

Proof. Let $h: \mathcal{H} \times[0,1] \rightarrow \mathcal{H}$ be an $\omega$-admissible deformation for $\mathscr{H}$. Fix $t_{0}$ such that $0<t_{0}<\omega(X)$. Fix $s$ such that $0<s<1$. Let $g: \mathcal{H} \rightarrow \mathcal{H}$ be as defined in the second part of the proof, p. 163, of 5.4 of [8]. Since $g(B)=\cup\{r(b): b \in B\}$ for each $b \in \mathcal{H}$, we have the following fact:

(1) If $B_{1}, B_{2} \in \mathcal{H}$ and $B_{1} \subset B_{2}$, then $g\left(B_{1}\right) \subset g\left(B_{2}\right)$. 
By 5.4 of [8], we can assume that $g$ is near enough to the identity map on $\mathcal{H}$ so that

(2) $\omega(g(X))>t_{0}$.

Since $h$ is an $\omega$-admissible deformation for $\mathcal{H}$ and since $s<1$ and $t_{0}>0, \omega(h(B, s))$ $<t_{0}$ for all $B \in \omega^{-1}\left(t_{0}\right)$. Thus, since $\omega^{-1}\left(t_{0}\right)$ is compact and since $h$ and $\omega$ are continuous, we have that

$$
\inf \left\{t_{0}-\omega(h(B, s)): B \in \omega^{-1}\left(t_{0}\right)\right\}>0 .
$$

Hence, by 5.4 of [8], we can assume that $g$ is near enough to the identity map on $\mathcal{H}$ so that

(3) $\omega(g(h(B, s)))<t_{0}$ for all $B \in \omega^{-1}\left(t_{0}\right)$.

By (1.2), there is a convex metric $d$ for $X, K_{d}$ maps $[0,+\infty) \times \mathcal{H}$ into $\mathcal{H}$, and $K_{d}$ is continuous. For each $B \in \omega^{-1}\left(t_{0}\right)$, let

$$
\sigma(B)=\left\{g\left(K_{d}(t, h(B, s))\right): t \geqslant 0\right\} .
$$

By the continuity of $K_{d}$ and $g$, we see that, for each $B \in \omega^{-1}\left(t_{0}\right), \sigma(B)$ is a subcontinuum of $\mathcal{H}$. Also, using (1), we see that, for each $B \in \omega^{-1}\left(t_{0}\right)$,

$$
g\left(K_{d}\left(t_{1}, h(B, s)\right)\right) \subset g\left(K_{d}\left(t_{2}, h(B, s)\right)\right) \text { if } 0 \leqslant t_{1} \leqslant t_{2} .
$$

Hence, by [18, Lemma 5 or $21,1.4], \sigma(B)$ is an order arc in $\mathcal{H}$ for each $B \in \omega^{-1}\left(t_{0}\right)$. Since $h, K_{d}$, and $g$ are continuous, it follows that $\sigma: \omega^{-1}\left(t_{0}\right) \rightarrow C(\mathcal{H})$ is continuous. For each $B \in \omega^{-1}\left(t_{0}\right)$, we have by (3) that

$$
\omega\left(g\left(K_{d}(0, h(B, s))\right)\right)<t_{0}
$$

and, letting $\delta=\operatorname{diam}_{d}(X)$, we have by (2) that

$$
\omega\left(g\left(K_{d}(\delta, h(B, s))\right)\right)>t_{0} .
$$

Hence, using the continuity of $K_{d}, g$, and $\omega$, we see that $\sigma(B) \cap \omega^{-1}\left(t_{0}\right) \neq \varnothing$ for each $B \in \omega^{-1}\left(t_{0}\right)$. Therefore, letting $\sigma_{t_{0}}: \omega^{-1}\left(t_{0}\right) \rightarrow \omega^{-1}\left(t_{0}\right)$ be as defined in (3.1) that $\sigma_{t_{0}}$ is continuous. By 5.4 of $[8], g(B) \not \supset A$ for any $B \in \mathcal{H}$. Thus, $\sigma_{t_{0}}$ maps $\omega^{-1}\left(t_{0}\right)$ into $\omega^{-1}\left(t_{0}\right)-\left\{B \in \omega^{-1}\left(t_{0}\right): B \supset A\right\}$. Also: for any given $\varepsilon>0$, it is easy to see that if $s$ is close enough to 1 and $g$ is near enough to the identity map on $\mathcal{H}$, then $\sigma_{t_{0}}$ is within $\varepsilon$ of the identity map on $\omega^{-1}\left(t_{0}\right)$. Therefore, $\left\{B \in \omega^{-1}\left(t_{0}\right): B \supset A\right\}$ is a $Z$-set in $\omega^{-1}\left(t_{0}\right)$.

The rest of this section is devoted to proving the following lemma. As in $[8, p$. 161], we let

$$
2_{A}^{X}=\left\{B \in 2^{X}: B \supset A\right\} .
$$

(3.3) Lemma. Let $X$ be a Peano continuum and let $A$ be a closed subset of $X$ such that $A$ contains an arc $J$ which is free in $X$. If $\omega$ is an admissible Whitney map for $2^{X}$, then, for each $t_{0}$ such that $0<t_{0}<\omega(X), 2{ }_{A}^{X} \cap \omega^{-1}\left(t_{0}\right)$ is a Z-set in $\omega^{-1}\left(t_{0}\right)$.

Proof. Let $h: 2^{X} \times[0,1] \rightarrow 2^{X}$ be an $\omega$-admissible deformation for $2^{X}$. Fix $t_{0}$ such that $0<t_{0}<\omega(X)$. Fix $s$ such that $0<s<1$. Since $h$ is an $\omega$-admissible deformation for $2^{X}$ and since $s<1$ and $t_{0}>0, \omega(h(B, s))<t_{0}$ for all $B \in \omega^{-1}\left(t_{0}\right)$. Since $\omega^{-1}\left(t_{0}\right)$. Since $\omega^{-1}\left(t_{0}\right)$ is compact and since $h$ and $\omega$ are continuous, it follows that

$(*) \inf \left\{t_{0}-\omega(h(B, s)): B \in \omega^{-1}\left(t_{0}\right)\right\}>0$. 
Let $d$ be a convex metric for $X$ (see (1.2)) and let $\delta=\operatorname{diam}_{d}(X)$. Let $g$ : $2^{X} \rightarrow 2^{X}-2_{A}^{X}$ be as defined in the proof on p. 162 of 5.4 of [8]. We will make significant use of the formula for $g$ later on. For now we note that if the diameter of the free $\operatorname{arc} J$ is small, then $g$ is near the identity map on $2^{X}$. Thus (since $A$ contains free arcs of arbitrarily small diameter), we can assume that $J$ is small enough so that $\omega(g(X))>t_{0}$ and, by using $(*)$, so that

$(* *) \omega(g(h(B, s)))<t_{0}$ for all $B \in \omega^{-1}\left(t_{0}\right)$.

Let us note if $L$ is a free arc in any continuum $M$ and if $I$ is a subarc of the interior $L^{\circ}$ of $L$, then $M-I^{\circ}$ has at most two components-furthermore, if $M$ is acyclic, $M-I^{\circ}$ has exactly two components. By (2.5), our continuum $X$ is contractible and, thus, $X$ is acyclic. Hence, by replacing $J$ with a subarc of $J^{\circ}$ if necessary, we can assume without loss of generality that $X-J^{\circ}$ has exactly two components $X_{1}$ and $X_{2}$. Note that $X_{i} \cap J$ is an end point of $J$ for each $i=1$ and 2. As in the proof of 5.4 of [8], we identify $J$ with the interval $[-1,1]$. We assume that the indexing of $X_{1}$ and $X_{2}$ was done so that $X_{1} \cap J=\{-1\}$ and $X_{2} \cap J=\{1\}$. We observe that if $x, y \in J$ such that $x \leqslant y$, then the restriction of $d$ to each of the sets $X_{1} \cup[-1, x],[x, y]$, and $[y, 1] \cup X_{2}$ is again a convex metric.

As in the proof of 5.4 of [8], we define $b^{+}$and $b^{-}$as follows. Let $B \in 2^{X}$. If $B \cap[0,1] \neq \varnothing$, then let

$$
b^{+}=\inf B \cap[0,1]
$$

and, if $B \cap[-1,0] \neq \varnothing$, then let

$$
b^{-}=\sup B \cap[-1,0] .
$$

If $Y$ is a subcontinuum of $X$, then let $K(\cdot, \cdot, Y)$ denote the restriction of $K_{d}$ in $(1.2)$ to $[0,+\infty) \times 2^{Y}$, i.e., for each $(t, E) \in[0,+\infty) \times 2^{Y}$,

$$
K(t, E ; Y)=\{y \in Y: d(y, z) \leqslant t \text { for some } z \in E\} .
$$

If the restriction of $d$ to $Y$ is a convex metric, then, by $(1.2), K(\cdot, \cdot, Y):[0,+\infty) \times 2^{Y}$ $\rightarrow 2^{Y}$ is continuous. For convenience in the formulas which will come later, we let

$$
K(t, \varnothing ; Y)=\varnothing
$$

for any $t \in[0,+\infty)$.

On the next few pages we will define a mapping $\psi$ from $[0, \delta] \times 2^{X}$ into $2^{X}-2_{A}^{X}$ such that for each $B \in 2^{X}, \psi(0, B)=g(B)$ and $\{\psi(t, B): 0 \leqslant t \leqslant \delta\}$ is an order arc in $2^{X}$. The complexity of the definition of $\psi$ is necessitated by the complexity and apparently unavoidable use of $g$ and by the requirement that $\psi(t, B) \notin 2_{A}^{X}$ for any $(t, B) \in[0, \delta] \times 2^{X}$. The continuity of $\psi$ depends on the continuity of the "growth" functions $K$, the continuity of $g$, and the "phasing out" of the growth inside $J$. We will not prove the continuity of $\psi$. In Diagrams 1 and 2 we illustrate two particular cases of the formula for $\psi$. These diagrams should be useful in understanding $\psi$ and the continuity of $\psi$. 
Let $(t, B) \in[0, \delta] \times 2^{X}$.

If $B \cap J=\varnothing$, then

$$
\psi(t, B)=K\left(t, B \cap X_{1} ; X_{1}\right) \cup K\left(t, B \cap X_{2} ; X_{2}\right)
$$

If $B \cap J \neq \varnothing$ and $B \cap J \subset[0,1]$, then

$$
\psi(t, B)=\left\{\begin{aligned}
K(t, & \left.B \cap X_{1} ; X_{1}\right) \\
& \cup K\left(t, B \cap\left(\left[b^{+}, 1\right] \cup X_{2}\right) ;\left[b^{+}, 1\right] \cup X_{2}\right) \\
& \cup K\left(t \cdot d\left(b^{+}, 1\right),\left\{2 b^{+}-1\right\} ; X_{1} \cup\left[-1,2 b^{+}-1\right]\right) \\
& \cup g(B), \text { if } \frac{1}{3} \leqslant b^{+} \leqslant 1, \\
K(t, & \left.B \cap X_{1} ; X_{1}\right) \\
& \cup K\left(t, g(B) \cap\left(\left[1-2 b^{+}, 1\right] \cup X_{2}\right) ;\left[1-2 b^{+}, 1\right] \cup X_{2}\right) \\
& \cup K\left(t \cdot d\left(b^{+}, 1\right),\left\{2 b^{+}-1\right\} ; X_{1} \cup\left[-1,2 b^{+}-1\right]\right),
\end{aligned}\right.
$$

If $B \cap J \neq \varnothing$ and $B \cap J \subset[-1,0]$, then $\psi(t, B)$ is defined in the symmetric fashion, using $b^{-}$instead of $b^{+}$.

If $B \cap[-1,0] \neq \varnothing, B \cap[0,1] \neq \varnothing$, and $-1 \leqslant b^{-} \leqslant-\frac{2}{3}$, then (see Diagram 1)

$$
\psi(t, B)=\left\{\begin{array}{c}
K\left(t, B \cap\left(X_{1} \cup\left[-1, b^{-}\right]\right) ; X_{1} \cup\left[-1, b^{-}\right]\right) \\
\cup K\left(t \cdot d\left(b^{-},-1\right),\left\{2 b^{-}+1\right\} ;\left[2 b^{-}+1,2 b^{+}-1\right]\right) \\
\cup K\left(t \cdot d\left(b^{+}, 1\right),\left\{2 b^{+}-1\right\} ;\left[2 b^{-}+1,2 b^{+}-1\right]\right) \\
\cup K\left(t, B \cap\left(\left[b^{+}, 1\right] \cup X_{2}\right) ;\left[b^{+}, 1\right] \cup X_{2}\right) \\
\cup g(B), \quad \text { if } \frac{1}{3} \leqslant b^{+} \leqslant 1, \\
K\left(t, B \cap\left(X_{1} \cup\left[-1, b^{-}\right]\right) ; X_{1} \cup\left[-1, b^{-}\right]\right) \\
\cup K\left(t \cdot d\left(b^{-},-1\right),\left\{2 b^{-}+1\right\} ;\left[2 b^{-}+1,2 b^{+}-1\right]\right) \\
\cup K\left(t \cdot d\left(b^{+}, 1\right),\left\{2 b^{+}-1\right\} ;\left[2 b^{-}+1,2 b^{+}-1\right]\right) \\
\cup K\left(t, g(B) \cap\left(\left[1-2 b^{+}, 1\right] \cup X_{2}\right) ;\left[1-2 b^{+}, 1\right] \cup X_{2}\right), \\
\quad \text { if } b^{-}+1 \leqslant b^{+} \leqslant \frac{1}{3}, \\
K\left(t, B \cap\left(X_{1} \cup\left[-1, b^{-}\right]\right) ; X_{1} \cup\left[-1, b^{-}\right]\right) \\
\cup K\left(t, g(B) \cap\left(\left[1-2 b^{+}, 1\right] \cup X_{2}\right) ;\left[1-2 b^{+}, 1\right] \cup X_{2}\right) \\
\cup g(B), \quad \text { if }\left(b^{-}+1\right) / 2 \leqslant b^{+} \leqslant b^{-}+1, \\
K\left(t, g(B) \cap\left(X_{1} \cup\left[-1,2 b^{+}-1\right]\right) ; X_{1} \cup\left[-1,2 b^{+}-1\right]\right) \\
\cup K\left(t, g(B) \cap\left(\left[1-2 b^{+}, 1\right] \cup X_{2}\right) ;\left[1-2 b^{+}, 1\right] \cup X_{2}\right), \\
\text { if } 0 \leqslant b^{+} \leqslant\left(1+b^{-}\right) / 2 .
\end{array}\right.
$$


If $B \cap[-1,0] \neq \varnothing, B \cap[0,1] \neq \varnothing$, and $-\frac{2}{3} \leqslant b^{-} \leqslant-\frac{1}{2}$, then

$$
\psi(t, B)=\left\{\begin{array}{c}
K\left(t, B \cap\left(X_{1} \cup\left[-1, b^{-}\right]\right) ; X_{1} \cup\left[-1, b^{-}\right]\right) \\
\cup K\left(t \cdot d\left(b^{-},-1\right),\left\{2 b^{-}+1\right\} ;\left[2 b^{-}+1,2 b^{+}-1\right]\right) \\
\cup K\left(t \cdot d\left(b^{+}, 1\right),\left\{2 b^{+}-1\right\} ;\left[2 b^{-}+1,2 b^{+}-1\right]\right) \\
\cup K\left(t, B \cap\left(\left[b^{+}, 1\right] \cup X_{2}\right) ;\left[b^{+}, 1\right] \cup X_{2}\right) \\
\cup g(B), \quad \text { if } b^{-}+1 \leqslant b^{+} \leqslant 1, \\
K\left(t, B \cap\left(X_{1} \cup\left[-1, b^{-}\right]\right) ; X_{1} \cup\left[-1, b^{-}\right]\right) \\
\cup K\left(t, B \cap\left(\left[b^{+}, 1\right] \cup X_{2}\right) ;\left[b^{+}, 1\right] \cup X_{2}\right) \\
\cup g(B), \quad \text { if } \frac{1}{3} \leqslant b^{+} \leqslant b^{-}+1, \\
K\left(t, B \cap\left(X_{1} \cup\left[-1, b^{-}\right]\right) ; X_{1} \cup\left[-1, b^{-}\right]\right) \\
\cup K\left(t, g(B) \cap\left(\left[1-2 b^{+}, 1\right] \cup X_{2}\right) ;\left[1-2 b^{+}, 1\right] \cup X_{2}\right) \\
\cup g(B), \quad \text { if }\left(b^{-}+1\right) / 2 \leqslant b^{+} \leqslant \frac{1}{3}, \quad \text { if } 0 \leqslant b^{+} \leqslant\left(b^{-}+1\right) / 2 . \\
K\left(t, g(B) \cap\left(X_{1} \cup\left[-1,2 b^{+}-1\right]\right) ; X_{1} \cup\left[-1,2 b^{+}-1\right]\right) \\
\cup K\left(t, g(B) \cap\left(\left[1-2 b^{+}, 1\right] \cup X_{2}\right) ;\left[1-2 b^{+}, 1\right] \cup X_{2}\right),
\end{array}\right.
$$

If $B \cap[-1,0] \neq \varnothing, B \cap[0,1] \neq \varnothing$, and $-\frac{1}{2} \leqslant b^{-} \leqslant-\frac{1}{3}$, then

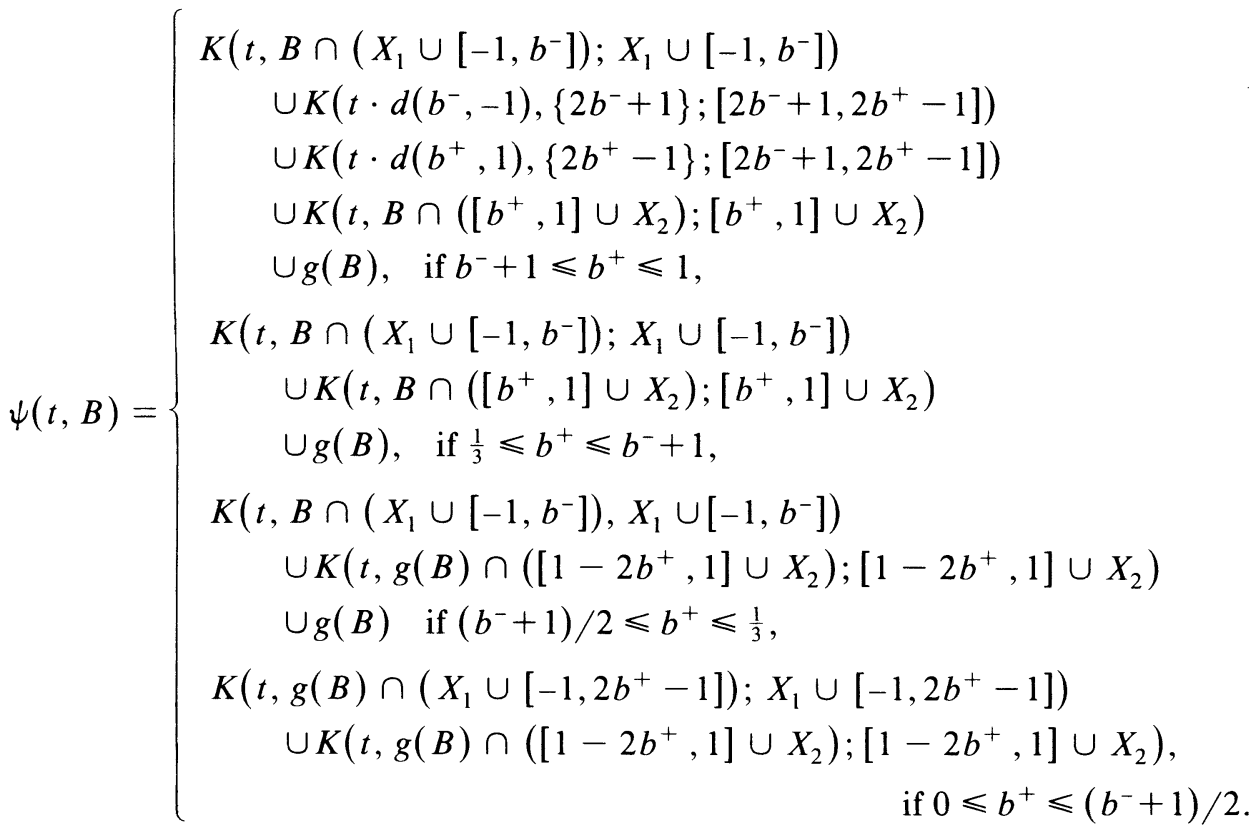

If $B \cap[-1,0] \neq \varnothing, B \cap[0,1] \neq \varnothing$, and $-\frac{1}{3} \leqslant b^{-} \leqslant 0$, then (see Diagram 2) 


$$
\psi(t, B)=\left\{\begin{array}{c}
K\left(t, g(B) \cap\left(X_{1} \cup\left[-1,-2 b^{-}-1\right]\right) ; X_{1} \cup\left[-1,-2 b^{-}-1\right]\right) \\
\cup K\left(t \cdot d\left(b^{-},-1\right),\left\{1+2 b^{-}\right\} ;\left[1+2 b^{-}, 2 b^{+}-1\right]\right) \\
\cup K\left(t \cdot d\left(b^{+}, 1\right),\left\{2 b^{+}-1\right\} ;\left[1+2 b^{-}, 2 b^{+}-1\right]\right) \\
\cup K\left(t, B \cap\left(\left[b^{+}, 1\right] \cup X_{2}\right) ;\left[b^{+}, 1\right] \cup X_{2}\right), \text { if } b^{-}+1 \leqslant b^{+} \leqslant 1, \\
K\left(t, g(B) \cap\left(X_{1} \cup\left[-1,-2 b^{-}-1\right]\right) ; X_{1} \cup\left[-1,-2 b^{-}-1\right]\right) \\
\cup K\left(t, B \cap\left(\left[b^{+}, 1\right] \cup X_{2}\right) ;\left[b^{+}, 1\right] \cup X_{2}\right) \\
\cup g(B), \text { if } 2 b^{-}+1 \leqslant b^{+} \leqslant b^{-}+1, \quad \text { if }-b^{-} \leqslant b^{+} \leqslant 1+2 b^{-}, \\
K\left(t, g(B) \cap\left(X_{1} \cup\left[-1,-2 b^{-}-1\right]\right) ; X_{1} \cup\left[-1,-2 b^{-}-1\right]\right) \\
\cup K\left(t, g(B) \cap\left(\left[1+2 b^{-}, 1\right] \cup X_{2}\right) ;\left[1+2 b^{-}, 1\right] \cup X_{2}\right), \\
K\left(t, g(B) \cap\left(X_{1} \cup\left[-1,2 b^{+}-1\right]\right) ; X_{1} \cup\left[-1,2 b^{+}-1\right]\right) \\
\cup K\left(t, g(B) \cap\left(\left[1-2 b^{+}, 1\right] \cup X_{2}\right) ;\left[1-2 b^{+}, 1\right] \cup X_{2}\right), \\
\quad \text { if } 0 \leqslant b^{+} \leqslant-b^{-} .
\end{array}\right.
$$

This completes the definition of $\psi$. To complete the proof of this lemma, we will use (3.1) in a manner similar to its use in the proof of (3.2). However, as we will see below, this involves several cases, depending on $\omega\left(X_{1}\right)$ and $\omega\left(X_{2}\right)$. For each such that $0 \leqslant s<1$, let $t_{1}(s)$ and $t_{2}(s)$ be defined as

$$
\begin{aligned}
& t_{1}(s)=\inf \left\{\omega(h(B, s)): B \in \omega^{-1}\left(t_{0}\right)\right\} ; \\
& t_{2}(s)=\sup \left\{\omega(h(B, s)): B \in \omega^{-1}\left(t_{0}\right)\right\} .
\end{aligned}
$$

Then for each $s$ such that $0 \leqslant s<1,0 \leqslant t_{1}(s) \leqslant t_{2}(s)<t_{0}$. We may assume that $J$ is small enough and $s$ is near enough to 1 so that for each $B \in \omega^{-1}\left(t_{0}\right), h(B, s) \cap X_{1} \neq$ $\varnothing$ or $h(B, s) \cap X_{2} \neq \varnothing$. We observe that if $h(B, s) \cap X_{i} \neq \varnothing$, then $\psi(\delta, h(B, s))$ $\supset X_{i}$, and if $h(B, s) \subset X_{i}$, then $\psi(\delta, h(B, s))=X_{i}(i=1$ or 2$)$. Thus

$(* * *)$ for each $B \in \omega^{-1}\left(t_{0}\right)$,

$$
\omega(\psi(\delta, h(B, s))) \geqslant \min \left\{\omega\left(X_{1}\right), \omega\left(X_{2}\right)\right\} .
$$

Furthermore, if $B \in \omega^{-1}\left(t_{0}\right)$ and if $h(B, s) \cap X_{i} \neq \varnothing$ for each $i=1$ and 2, then $\omega(\psi(\delta, h(B, s))) \geqslant \omega\left(X_{1} \cup X_{2}\right)=\omega\left(X-J^{\circ}\right)=\omega(g(X))>t_{0}$. For each $B \in$ $\omega^{-1}\left(t_{0}\right)$, let

$$
\sigma(B)=\{\psi(t, h(B, s)): 0 \leqslant t \leqslant \delta\}
$$

Since $h$ and $\psi$ are continuous, $\sigma(B)$ is a subcontinuum of $2^{X}$. It follows from the definition of $\psi$ that if $B \in \omega^{-1}\left(t_{0}\right)$ and $0 \leqslant t^{\prime} \leqslant t^{\prime \prime} \leqslant \delta$, then $\psi\left(t^{\prime}, h(B, s)\right) \subset$ $\psi\left(t^{\prime \prime}, h(B, s)\right)$. Hence, by Lemma 5 of $[\mathbf{1 8}], \sigma(B)$ is an order arc in $2^{X}$ for each $B \in \omega^{-1}\left(t_{0}\right)$. Since $h$ and $\psi$ are continuous, it follows that $\sigma: \omega^{-1}\left(t_{0}\right) \rightarrow C\left(2^{X}\right)$ is continuous. For each $B \in \omega^{-1}\left(t_{0}\right)$, we have by $(* *)$ that $\omega(\psi(0, h(B, s)))=$ $\omega(g(h(B, s)))<t_{0}$. 


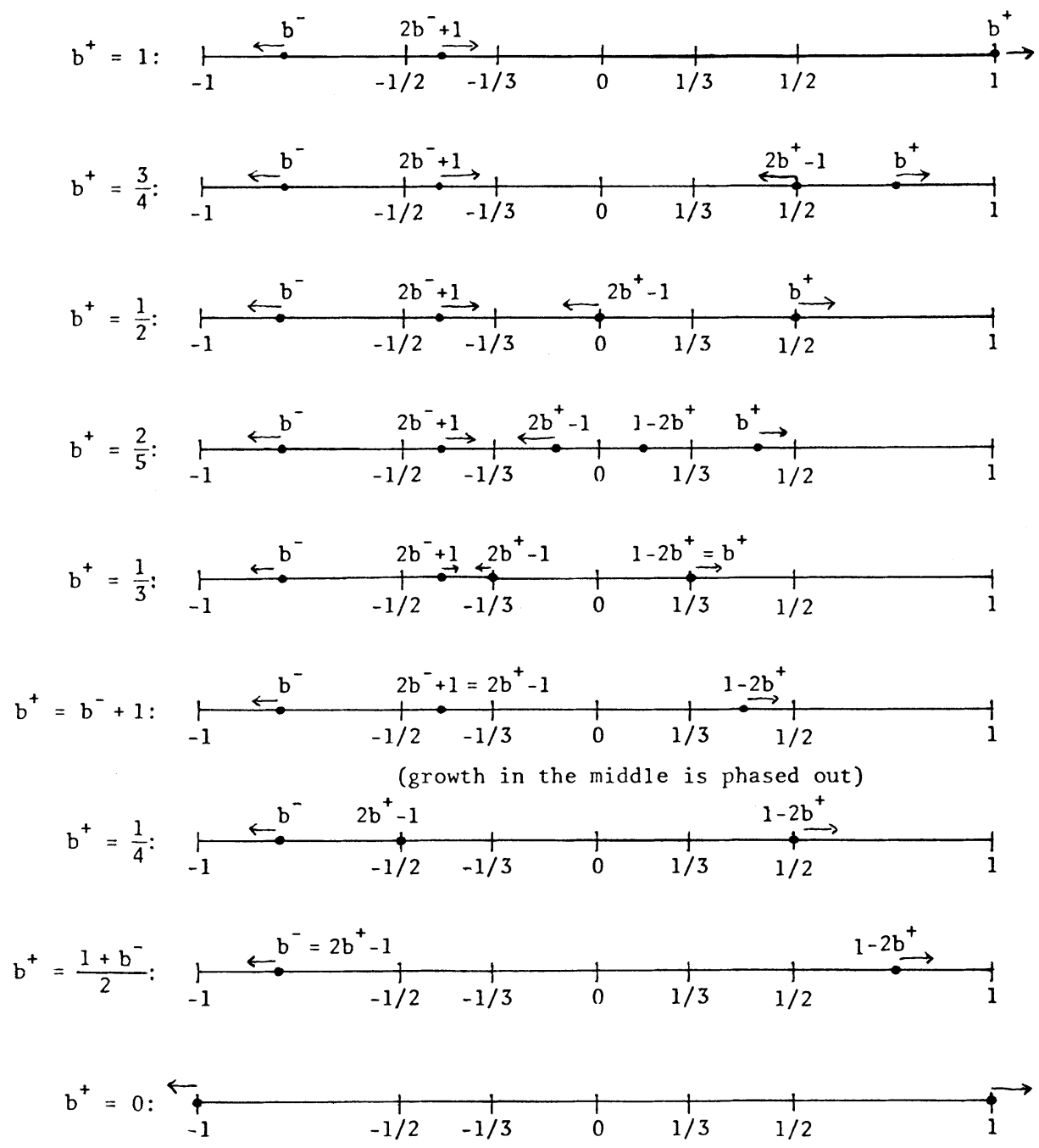

Diagram 1 .

This diagram is for a fixed $b^{-}$such that $-<b^{-}<-2 / 3$ and selected values of $b^{+}$; the arrows indicate the directions in which "growth" is allowed; the dots represent points belonging to $g(B)$.

Case I. Assume that $\omega\left(X_{1}\right) \geqslant t_{0}$ and $\omega\left(X_{2}\right) \geqslant t_{0}$. Then, by $(* * *)$, for each $B \in \omega^{-1}\left(t_{0}\right), \omega(\psi(\delta, h(B, s))) \geqslant t_{0}$. It follows that for each $B \in \omega^{-1}\left(t_{0}\right), \sigma(B) \cap$ $\omega^{-1}\left(t_{0}\right) \neq \varnothing$. Hence, letting $\sigma_{t_{0}}: \omega^{-1}\left(t_{0}\right) \rightarrow \omega^{-1}\left(t_{0}\right)$ be as defined in (3.1), we have by (3.1) that $\sigma_{t_{0}}$ is continuous.

Case II. Assume that $\omega\left(X_{1}\right) \geqslant t_{0}$ and $\omega\left(X_{2}\right)<t_{0}$. Let $v \in J^{\circ}$ such that $t_{3}=$ $\omega\left([v, 1] \cup X_{2}\right)<t_{0}$. Choose $s^{\prime}$ so that $s \leqslant s^{\prime} \leqslant 1$ and $t_{3}<t_{1}\left(s^{\prime}\right) \leqslant t_{2}\left(s^{\prime}\right)<t_{0}$. Let $J^{\prime}=[v, 1], X_{1}^{\prime}=X_{1} \cup[-1, v]$, and $X_{2}^{\prime}=X_{2}$, and define $g^{\prime}, \psi^{\prime}$, and $\sigma^{\prime}$ in the analogous fashion, depending on $J^{\prime}$ instead of $J$. If $B \in \omega^{-1}\left(t_{0}\right)$, then $h\left(B, s^{\prime}\right) \cap X_{1}^{\prime} \neq \varnothing$ 


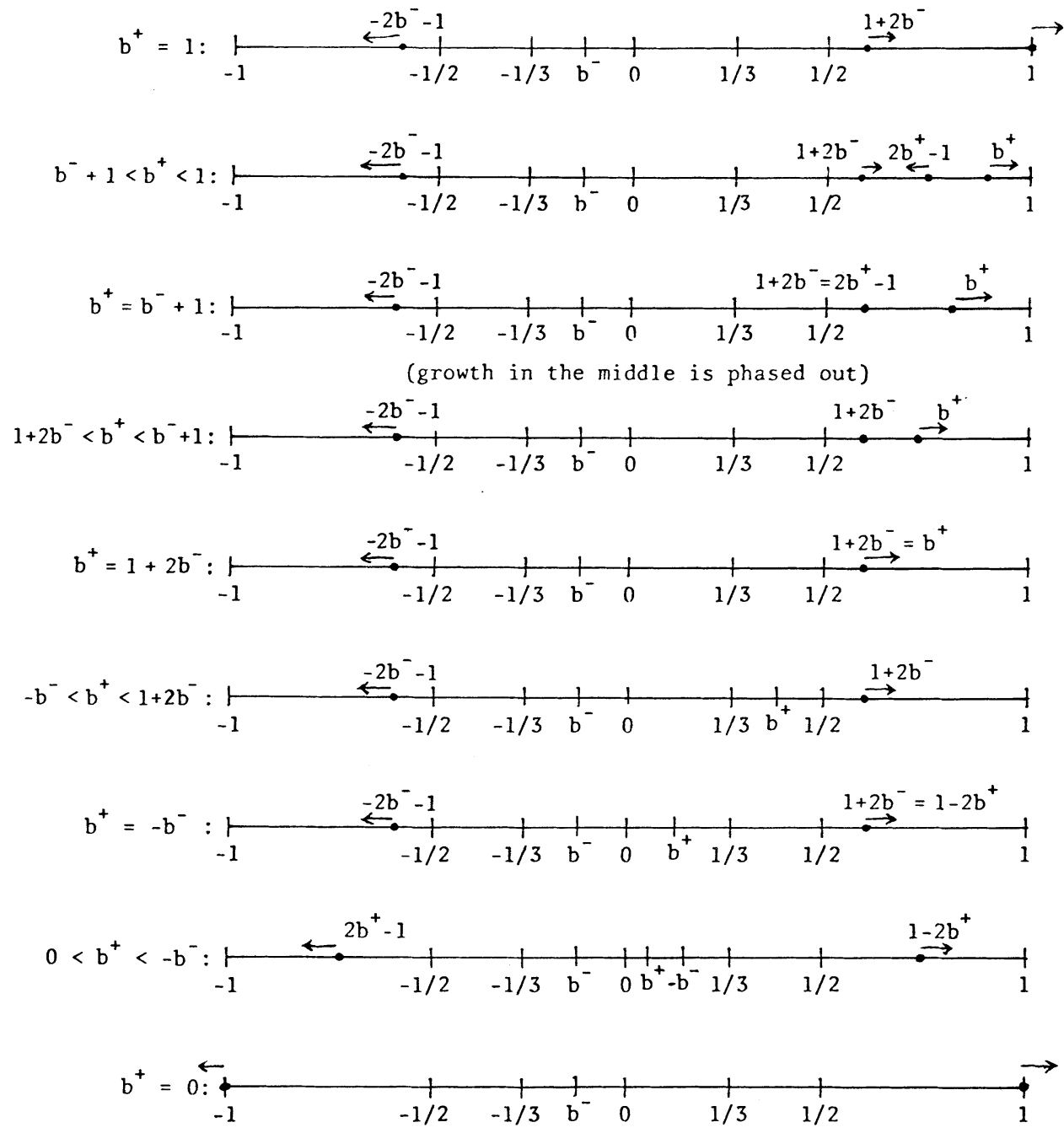

Diagram 2.

This diagram is for a fixed $b^{-}$such that $-1 / 3<b^{-}<0$ and selected values of $b^{+}$; the arrows indicate the directions in which "growth" is allowed; the dots represent points belonging to $g(B)$.

or $h\left(B, s^{\prime}\right) \cap X_{2}^{\prime} \neq \varnothing$. Furthermore, since $\omega\left([v, 1] \cup X_{2}^{\prime}\right)=t_{3}<t_{1}\left(s^{\prime}\right)$, if $h\left(B, s^{\prime}\right) \cap$ $X_{2}^{\prime} \neq \varnothing$, then $h\left(B, s^{\prime}\right) \cap X_{1}^{\prime} \neq \varnothing$. It follows that for each $B \in \omega^{-1}\left(t_{0}\right)$,

$$
\omega\left(\psi^{\prime}\left(\delta, h\left(B, s^{\prime}\right)\right)\right) \geqslant \omega\left(X_{1}^{\prime}\right)>t_{0} .
$$

Thus, for each $B \in \omega^{-1}\left(t_{0}\right), \sigma^{\prime}(B) \cap \omega^{-1}\left(t_{0}\right) \neq \varnothing$. Hence, letting $\sigma_{t_{0}^{\prime}}: \omega^{-1}\left(t_{0}\right) \rightarrow$ $\omega^{-1}\left(t_{0}\right)$ be as defined in (3.1), we have by (3.1) that $\sigma_{t_{0}}^{\prime}$ is continuous.

Case III. Assume that $\omega\left(X_{1}\right)<t_{0}$ and $\omega\left(X_{2}\right) \geqslant t_{0}$. The details in this case are analogous to those in Case II. 
Case IV. Assume that $\omega\left(X_{1}\right)<t_{0}$ and $\omega\left(X_{2}\right)<t_{0}$. If for some $v \in J^{\circ}$,

$$
\omega\left(X_{1} \cup[-1, v]\right) \geqslant t_{0},
$$

we proceed as in Case II (treating [v,1] as $J$ ). So we assume that $\omega\left(X_{1} \cup J\right) \leqslant t_{0}$. Choose $v_{1} \in J^{\circ}$ so that $\omega\left(\left[v_{1}, 1\right] \cup X_{2}\right)<t_{0}$ and choose $v_{2} \in J^{\circ}$ such that $v_{1}<v_{2}<$ 1. Let $t_{3}=\omega\left(X_{1} \cup\left[-1, v_{2}\right]\right)$ and $t_{4}=\omega\left(\left[v_{1}, 1\right] \cup X_{2}\right)$. Then $\max \left\{t_{3}, t_{4}\right\}<t_{0}$. Choose $s^{\prime}$ so that $s \leqslant s^{\prime}<1$ and $\max \left\{t_{3}, t_{4}\right\}<t_{1}\left(s^{\prime}\right) \leqslant t_{2}\left(s^{\prime}\right)<t_{0}$. Let $J^{\prime}=$ $\left[v_{1}, v_{2}\right], X_{1}^{\prime}=X_{1} \cup\left[-1, v_{1}\right]$, and $X_{2}^{\prime}=\left[v_{2}, 1\right] \cup X_{2}$, and define $g^{\prime}, \psi^{\prime}$, and $\sigma^{\prime}$ in the analogous fashion, depending on $J^{\prime}$ instead of $J$. If $B \in \omega^{-1}\left(t_{0}\right)$, then $h\left(B, s^{\prime}\right) \cap X_{1}^{\prime}$ $\neq \varnothing$ and $h\left(B, s^{\prime}\right) \cap X_{2}^{\prime} \neq \varnothing$, and hence

$$
\omega\left(\psi^{\prime}\left(\delta, h\left(B, s^{\prime}\right)\right)\right) \geqslant \omega\left(X_{1}^{\prime} \cup X_{2}^{\prime}\right)>t_{0} .
$$

Thus, for each $B \in \omega^{-1}\left(t_{0}\right), \sigma^{\prime}(B) \cap \omega^{-1}\left(t_{0}\right) \neq \varnothing$. Hence, letting $\sigma_{t_{0}}^{\prime}: \omega^{-1}\left(t_{0}\right) \rightarrow$ $\omega^{-1}\left(t_{0}\right)$ be as defined in (3.1), we have by (3.1) that $\sigma_{t_{0}^{\prime}}^{\prime}$ is continuous.

Since $\psi\left(\right.$ or $\left.\psi^{\prime}\right)$ maps $[0, \delta] \times 2^{X}$ into $2^{X}-2_{A}^{X}$, it follows that $\sigma_{t_{0}}\left(\right.$ or $\left.\sigma_{t_{0}}^{\prime}\right)$ maps $\omega^{-1}\left(t_{0}\right)$ into $\omega^{-1}\left(t_{0}\right)-\left\{B \in \omega^{-1}\left(t_{0}\right): B \supset A\right\}$. Finally, in each of the above cases, for any given $\varepsilon>0$, if $s$ (or $s^{\prime}$ ) is close enough to 1 and $g$ (or $g^{\prime}$ ) is near enough to the identity map on $2^{X}$, then $\sigma_{t_{0}}\left(\right.$ or $\sigma_{t_{0}}^{\prime}$ ) is within $\varepsilon$ of the identity map on $\omega^{-1}\left(t_{0}\right)$. Therefore, $\left\{B \in \omega^{-1}\left(t_{0}\right): B \supset A\right\}$ is a $Z$-set in $\omega^{-1}\left(t_{0}\right)$. This completes the proof of (3.3).

4. Main results. Our most general result concerning when positive Whitney levels are Hilbert cubes is (4.1). Its proof uses (2.9), Torunczyk's Theorem stated in (1.1), and the three results in $\S 3$. A companion theorem to (4.1) is in (4.10).

(4.1) Theorem. Let $X$ be a Peano continuum. If there is an admissible Whitney map $\omega$ for $2^{X}$, then $\omega^{-1}\left(t_{0}\right)$ is a Hilbert cube whenever $0<t_{0}<\omega(X)$. If there is an admissible Whitney map $\omega$ for $C(X)$ and if $X$ contains no free arc, then $\omega^{-1}\left(t_{0}\right)$ is a Hilbert cube whenever $0<t_{0}<\omega(X)$.

Proof. Let $\mathcal{H}=2^{X}$ or $C(X)$, let $h: \mathcal{K} \times[0,1] \rightarrow \mathcal{H}$ be an $\omega$-admissible deformation for $\mathcal{H}$, and fix $t_{0}$ such that $0<t_{0}<\omega(X)$. By (2.9), $\omega^{-1}\left(t_{0}\right)$ is an absolute retract. Thus, it suffices to show that $\omega^{-1}\left(t_{0}\right)$ satisfies the second part of (1.1). By (1.2), there is a convex metric $d$ for $X, K_{d}$ maps $[0,+\infty) \times \mathcal{H}$ into $\mathcal{H}$, and $K_{d}$ is continuous. Fix $s$ such that $0<s<1$. Define $\sigma$ on $\omega^{-1}\left(t_{0}\right)$ by letting

$$
\sigma(B)=\left\{K_{d}(t, h(B, s)): t \geqslant 0\right\}
$$

for each $B \in \omega^{-1}\left(t_{0}\right)$. It follows easily from the properties of $h$ and $K_{d}$ (compare proof of (3.2)) that $\sigma$ is a continuous function from $\omega^{-1}\left(t_{0}\right)$ into $C(\mathcal{H})$ and that, for each $B \in \omega^{-1}\left(t_{0}\right), \sigma(B)$ is an order arc in $\mathcal{H}$ such that $\sigma(B) \cap \omega^{-1}\left(t_{0}\right) \neq \varnothing$. Hence, letting $\sigma_{t_{0}}: \omega^{-1}\left(t_{0}\right) \rightarrow \omega^{-1}\left(t_{0}\right)$ be as defined in (3.1), we have by (3.1) that $\sigma_{t_{0}}$ is continuous. We will show that $\sigma_{t_{0}}$ is a $Z$-map by using (3.2), (3.3), and modifications of ideas in [25, p. 39]. Since $h$ is an $\omega$-admissible deformation for $\mathcal{H}$ and since $s<1$ and $t_{0}>0, \omega(h(B, s))<t_{0}$ for all $B \in \omega^{-1}\left(t_{0}\right)$. Thus, since $\omega^{-1}\left(t_{0}\right)$ is compact and since $h$ and $\omega$ are continuous, we see that

(\#) $\sup \left\{\omega(h(B, s)): B \in \omega^{-1}\left(t_{0}\right)\right\}<t_{0}$. 
From the definition of $\sigma_{t_{0}}$ we have that, for each $B \in \omega^{-1}\left(t_{0}\right)$, there exists $t_{B}$ such that $\sigma_{t_{0}}(B)=K_{d}\left(t_{B}, h(B, s)\right)$. Since $\omega\left(\sigma_{t_{0}}(B)\right)=t_{0}$ for all $B \in \omega^{-1}\left(t_{0}\right)$, it follows easily from (\#) that there exists $\gamma>0$ such that $t_{B} \geqslant \gamma$ for all $B \in \omega^{-1}\left(t_{0}\right)$. Let $\left\{p_{1}, \ldots, p_{n}\right\}$ be a $\gamma / 2$-net for $X$, i.e., $\left\{p_{1}, \ldots, p_{n}\right\}$ is a finite subset of $X$ such that any point of $X$ is within $\gamma / 2$ of some $p_{i}$. For each $i=1, \ldots, n$, let

$$
\mathscr{P}_{i}=\left\{E \in \omega^{-1}\left(t_{0}\right): E \supset K_{d}\left(\gamma / 2,\left\{p_{i}\right\}\right)\right\} .
$$

We now show that $\sigma_{t_{0}}\left[\omega^{-1}\left(t_{0}\right)\right] \subset \cup_{i=1}^{n} \mathscr{P}_{i}$. Let $B_{0} \in \omega^{-1}\left(t_{0}\right)$. Let $x_{0} \in h\left(B_{0}, s\right)$. Then, there exists $p_{j}$ such that $d\left(x_{0}, p_{j}\right) \leqslant \gamma / 2$. For any point $y \in K_{d}\left(\gamma / 2,\left\{p_{j}\right\}\right)$, we have

$$
d\left(y, x_{0}\right) \leqslant d\left(y, p_{j}\right)+d\left(p_{j}, x_{0}\right) \leqslant \gamma \leqslant t_{B_{0}}
$$

and, thus, $K_{d}\left(\gamma / 2,\left\{p_{j}\right\}\right) \subset K_{d}\left(t_{B_{0}}, h\left(B_{0}, s\right)\right)=\sigma_{t_{0}}\left(B_{0}\right)$. Hence, we have proved that $\sigma_{t_{0}}\left[\omega^{-1}\left(t_{0}\right)\right] \subset \cup_{i=1}^{n} \mathscr{P}_{i}$. By (3.2) and (3.3), each $\mathscr{P}_{i}$ is a $Z$-set in $\omega^{-1}\left(t_{0}\right)$. Hence, $\sigma_{t_{0}}\left[\omega^{-1}\left(t_{0}\right)\right]$ is contained in a finite union of $Z$-sets in $\omega^{-1}\left(t_{0}\right)$. Thus, $\sigma_{t_{0}}$ is a $Z$-map. It is easy to see that if $s$ had been sufficiently close to 1 , then $\sigma_{t_{0}}$ would have been as near the identity map on $\omega^{-1}\left(t_{0}\right)$ as desired. Hence, we have proved that the identity map on $\omega^{-1}\left(t_{0}\right)$ is a uniform limit of $Z$-maps. Therefore, by $(1.1), \omega^{-1}\left(t_{0}\right)$ is a Hilbert cube and we have proved (4.1).

The following corollary will be used in (4.3) through (4.5) to show that a number of properties are not strongly Whitney-reversible.

(4.2) CoROLLARY. If $X$ is the (topological) cone over any nonempty compact locally connected metric space $Y$, then there is a Whitney map $\omega$ for $2^{X}$ such that $\omega^{-1}\left(t_{0}\right)$ is a Hilbert cube whenever $0<t_{0}<\omega(X)$. In addition, if each component of $Y$ consists of more than one point, there is such a Whitney map $\mu$ for $C(X)$.

Proof. Use (2.15) and (4.1).

Recall from $\$ 1$ the definition of a strong Whitney-reversible property and, from $\left[2\right.$, p. 30], the definitions of local $n$-connectedness and $\mathrm{LC}^{n}$ for $n=0,1,2, \ldots, \infty$. In [22, Theorem 4] it was shown that local 0 -connectedness is strongly Whitney-reversible. Thus, it is natural to wonder if the higher degrees of local connectedness are strongly Whitney-reversible. In 14.57 of [21] it was asked if the property of being an absolute retract and if the property of being an absolute neighborhood retract are strongly Whitney-reversible. The following easy consequence of (4.2) shows that these properties, as well as many other properties, are not strongly Whitney-reversible.(see (4.4)).

(4.3) THeOREM. If $P$ is a topological property which the Hilbert cube has but which the cone over some Peano continuum does not have, then P is not a strong Whitney-reversible property.

Proof. Assume that $X$, the cone over some Peano continuum, does not have property $P$. Then use the Whitney map $\mu$ for $C(X)$ guaranteed by the second part of (4.2). 
(4.4) COROLlaRY. The following properties are not strongly Whitney-reversible: Being an absolute retract, being an absolute neighborhood retract, being locally $n$-connected for any $n \geqslant 1$, being $L C^{n}$ for any $n=1,2, \ldots, \infty$, being locally contractible, being homogeneous, and having the fixed point property.

Proof. Apply (4.3) to the cones over various examples in [2, pp. 30-31 and 14].

(4.5) REMARKS. By recalling the proof of (4.3), we can obtain some information not explicitly stated in (4.4). For example, let $X$ be the cone over a one-dimensional Peano continuum which is not locally contractible; then, using the proof of (4.3), we see that the property of being an absolute retract is not strongly Whitney-reversible even for two-dimensional continua $X$. In connection with this fact, we note that being an absolute retract is strongly Whitney reversible for one-dimensional continua (use [22, Theorem 4; 23, 4.3 and 2, 13.5, p. 138]). Now let $X$ be a continuum such that for some Whitney map $\omega$ for $2^{X}$ or $C(X), \omega^{-1}(t)$ is an absolute retract for all $0<t<\omega(X)$. Then, using ideas in the proof of (2.11), we see that $X$ has trivial shape. Hence, if $X$ is an absolute neighborhood retract, $X$ is an absolute retract. Thus, a finite-dimensional continuum which is used to show that being an absolute retract is not strongly Whitney-reversible must fail to be locally contractible (by $[2,10.4$, p. 122]). However, by applying the proof of (4.3) to the cone over the continuum in 11.1 of [2, p. 124], we see that the property of being an absolute retract is not strongly Whitney-reversible for locally contractible continua in general. Finally, noting that all of our examples for (4.4) are cones and, hence, are contractible, we mention that contractibility is not Whitney-reversible [23, 3.7]. We do not know if contractibility is Whitney-reversible or strongly Whitney-reversible for Peano continua.

The following theorem will be used in (4.7) to answer some questions in [21].

(4.6) THEOREM. If a Peano continuum X can be embedded in a Banach space so as to be starshaped, then there is an admissible Whitney map $\omega$ for $2^{X}$ such that $\omega^{-1}\left(t_{0}\right)$ is a Hilbert cube whenever $0<t_{0}<\omega(X)$. In addition, if $X$ contains no free arc, there is such a Whitney map $\mu$ for $C(X)$.

Proof. Use (2.2), (2.14), and (4.1).

(4.7) Remarks. It is of interest to know when all Whitney levels in $C(X)$ are homeomorphic (see [21, pp. 433-441]). A continuum $X$ is said to be weakly Whitney stable (resp., Whitney stable) provided that for some (resp., every) Whitney map for $C(X)$, all Whitney levels below $\{X\}$ are homeomorphic [21, p. 434]. Let $X$ be the Hilbert cube. Then, the existence of $\mu$ in (4.6) shows that the Hilbert cube is weakly Whitney stable. In 14.42.1 of [21] it was shown that the Hilbert cube is not Whitney stable. Thus, these two types of stability are not equivalent, which answers a question in 14.42 .2 of [21]. Since there are subcontinua of the Hilbert cube which are not weakly Whitney stable (e.g., a 2-cell), we see that the property of being weakly Whitney stable is not hereditary. This answers a question in 14.42.3 of [21]. Now let $X$ be any compact convex subset of a Banach space such that $\operatorname{dim}(X) \geqslant 2$. In 14.38 of [21] it was asked if $\omega^{-1}(t)$ is a Hilbert cube for all $0<t<\omega(X)$ when $\omega$ is the Whitney map for $C(X)$ defined in [28, p. 275]. By recalling the proofs of (2.13), 
(2.14), and (4.6), we see that we have answered this question affirmatively and that we have shown the same is true for $2^{X}$ (even if $\operatorname{dim}(X)=1$ ).

It is known that if $X$ is dendrite, then $2^{X}$ is a Hilbert cube and, if the branch points of $X$ are dense in $X, C(X)$ is a Hilbert cube (see [7 and 27], resp.). The following theorem is an analogue for Whitney levels of these results.

(4.8) THEOREM. If $X$ is a dendrite, then there is a Whitney map $\omega$ for $2^{X}$ such that $\omega^{-1}\left(t_{0}\right)$ is a Hilbert cube whenever $0<t_{0}<\omega(X)$. If $X$ is a dendrite such that the branch points of $X$ are dense in $X$, then, for any Whitney map $\mu$ for $C(X), \mu^{-1}\left(t_{0}\right)$ is a Hilbert cube whenever $0<t_{0}<\mu(X)$.

Proof. The first part of the theorem follows from (2.16) and (4.1). The second part of the theorem follows from (2.17) and (4.1).

(4.9) REMARK. We do not know if the first part of (4.8) is true for every Whitney map for $2^{X}$. In particular, we do not know if every Whitney map for $2^{X}$ is admissible when $X$ is a dendrite or, more generally, when $X$ is a smooth dendroid (compare (2.17)).

The following result is an analogue of (4.1) for the "bottoms" and "tops" of hyperspaces. The proof uses West's compactification theorem [27]. The part of the theorem concerning "tops" can also be proved using 5.1 of [6].

(4.10) TheOrEM. Let $X$ be a Peano continuum. If there is an admissible Whitney map $\omega$ for $2^{X}$, then $\omega^{-1}\left(\left[0, t_{0}\right]\right)$ and $\omega^{-1}\left(\left[t_{0}, \omega(X)\right]\right)$ are Hilbert cubes whenever $0<t_{0}<\omega(X)$. If there is an admissible Whitney map $\omega$ for $C(X)$ and if $X$ contains no free arc, then $\omega^{-1}\left(\left[0, t_{0}\right]\right)$ and $\omega^{-1}\left(\left[t_{0}, \omega(X)\right]\right)$ are Hilbert cubes whenever $0<t_{0}<$ $\omega(X)$.

Proof. Let $\mathcal{H}=2^{X}$ or $C(X)$ and let $h: \mathcal{H} \times[0,1] \rightarrow \mathscr{H}$ be an $\omega$-admissible deformation for $\mathcal{H}$. Fix $t_{0}$ such that $0<t_{0}<\omega(X)$, let $\mathscr{Q}=\omega^{-1}\left(\left[0, t_{0}\right]\right)$ or $\omega^{-1}\left(\left[t_{0}, \omega(X)\right]\right)$, and let $\mathscr{B}=\mathbb{Q}-\omega^{-1}\left(t_{0}\right)$. By (2.12), $\omega$ is an open map. Hence, by using $[29,4.32$, p. 130], it follows that $\mathcal{Q}$ is the closure in $\mathcal{H}$ of $\mathscr{B}$. Thus, we may consider $\mathscr{Q}$ to be a compactification of $\Re$. The remainder in the compactification is $\omega^{-1}\left(t_{0}\right)$ which, by (4.1), is a Hilbert cube. By [7], $\mathcal{H}$ is a Hilbert cube. Thus, since $\mathscr{B}$ is an open subset of $\mathcal{H}, \mathscr{B}$ is a Hilbert cube manifold [5, p. 18]. It follows easily using (2.9) that $\mathcal{Q}$ is a retract of $\mathcal{H}$. Hence, $\mathcal{Q}$ is an absolute retract. Thus, by combining Edwards' Theorem [5, 44.1, p. 106] with a result of Chapman [5, 22.1, p. 36], we have that $\mathscr{Q}$ is a Hilbert cube factor. If $\mathscr{Q}=\omega^{-1}\left(\left[0, t_{0}\right]\right)$, then, using $h \mid \mathcal{Q}$ and (ii) of (2.1), we see easily that $\omega^{-1}\left(t_{0}\right)$ is a $Z$-set in $\mathscr{Q}$; if $\mathscr{Q}=\omega^{-1}\left(\left[t_{0}, \omega(X)\right]\right)$, then, using $K_{d} \mid[0,+\infty) \times \mathscr{Q}$ where $d$ and $K_{d}$ are as in (1.2), we see easily that $\omega^{-1}\left(t_{0}\right)$ is a $Z$-set in $Q$. Our theorem now follows from Theorem 1 of [27, p. 605].

(4.11) Remark. By using (4.10) instead of (4.1) in the proof of (4.2), (4.6), and (4.8), we see that these three results for levels also hold for the "bottoms" and "tops" of the hyperspaces.

(4.12) REMARK. In connection with (4.10) we note the following fact. Let $Q$ denote the Hilbert cube and let id: $Q \rightarrow Q$ denote the identity map. Let $X$ be a Peano continuum. Let $\mathcal{H}=2^{X}$ or, if $X$ contains no free arc, let $\mathcal{H}=C(X)$. If $\omega$ is an 
admissible Whitney map for $\mathcal{H}$, then

$$
\omega \times \text { id: } \mathcal{H} \times Q \rightarrow[0, \omega(X)] \times Q
$$

is an open near-homeomorphism. This fact follows immediately from (2.12), [7], and 43.1 of $[5$, p. 103].

In [10] some geometric models are constructed for $C(X)$ when $X$ is not locally connected by using results about intervals of continua [9] and results from infinitedimensional topology. By using theorems in this section and techniques in [10], some geometric models can be constructed for Whitney levels in $C(X)$ and $2^{X}$ when $X$ is not locally connected. For example, letting $X$ be the continuum drawn below, the authors have constructed geometric models for each Whitney level in $C(X)$ using the Whitney map in [28, p. 275]. One can see from these models that each positive Whitney level is arc-smooth (for definition, see [12]). Thus, arc-smoothness is not strongly Whitney-reversible since $X$ is not even contractible. It is known that contractibility is not Whitney-reversible [23,3.7]; it would be of interest to know if arc-smoothness is Whitney-reversible.

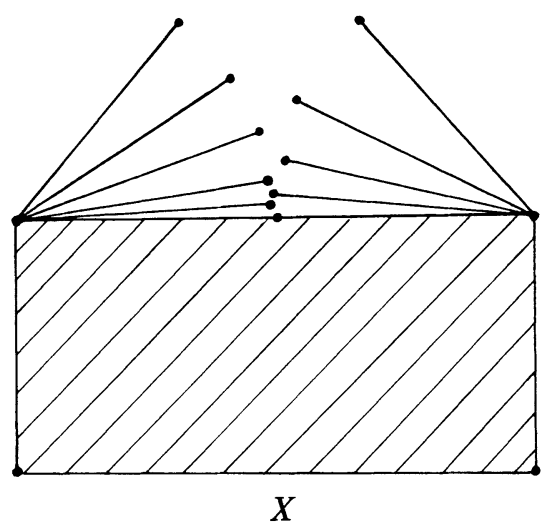

We conclude this paper with some questions concerning the material in $\S 2$.

By examining the proofs of (2.13)-(2.16), (4.2), (4.6), and (4.8), it can be seen that the Whitney maps $\omega$ and $\mu$ in (4.2), (4.6) and (4.8) can be chosen so that $\mu=\omega \mid C(X)$.

(4.13) Questions. Is the restriction to $C(X)$ of every admissible Whitney map for $2^{X}$ an admissible Whitney map for $C(X)$ ? Can every admissible Whitney map for $C(X)$ be extended to an admissible Whitney map for $2^{X}$ (compare [26])? If there is an admissible Whitney map for one of the hyperspaces $2^{X}$ or $C(X)$, is there an admissible Whitney map for the other hyperspaces?

Recalling the results in (2.4), (2.5) and (2.11), we ask the following question.

(4.14) Question. If there is an admissible Whitney map for $2^{X}$ or $C(X)$, then must $X$ be contractible?

Finally, we remark that it would be of interest to determine when Whitney levels are Hilbert cube manifolds. The following example indicates some of the subtleties involved with this problem. 
(4.15) ExAmple. Let $X$ denote the unit circle in the plane, let $d$ denote the arc length metric for $X$ (note that $d$ is a convex metric), and let $\omega$ be the Whitney map for $2^{X}$ used in the proof of (2.13). Let $x_{1}=(1,0), x_{2}=(-1 / 2, \sqrt{3 / 2})$, and $x_{3}=$ $(-1 / 2,-\sqrt{3 / 2})$. Let $A_{0}=\left\{x_{1}, x_{2}, x_{3}\right\}$, and let $t_{0}=\omega\left(A_{0}\right)$. For each $i=1,2$, and 3 , let $U_{i}=\left\{x \in X: d\left(x, x_{i}\right)<\pi / 6\right\}$. Let $\mathcal{Q}=\left\{B \in 2^{X}: B \subset \cup_{i=1}^{3} U_{i}\right.$ and $B \cap U_{i} \neq \varnothing$ for each $i=1,2$, and 3$\}$. Note that $\mathscr{U}$ is an open subset of $2^{X}$. By using the formula for $\omega$, we obtain the following fact: If $B \in \mathcal{Q}$ and $B$ is a three-point set, then $\omega(B) \geqslant t_{0}$. Hence, $\Omega \cap \omega^{-1}\left(t_{0}\right)$ consists entirely of three-point sets. Thus, $Q \cap \cap$ $\omega^{-1}\left(t_{0}\right)$ is a finite-dimensional nonempty open subset of $\omega^{-1}\left(t_{0}\right)$. Therefore, even in this simple case and for this nice Whitney map for $2^{X}, \omega^{-1}\left(t_{0}\right)$ is not a Hilbert cube manifold.

\section{REFERENCES}

1. R. H. Bing, Partitioning a set, Bull. Amer. Math. Soc. 55 (1949), 1101-1110.

2. K. Borsuk, Theory of retracts, Monogr. Mat. 44 (1967).

3. C. E. Capel and W. L. Strother, Multi-valued functions and partial order, Portugal. Math. 17 (1958), 41-47.

4. J. H. Carruth, $A$ note on partially ordered compacta, Pacific J. Math. 24 (1968), 229-231.

5. T. A. Chapman, Lectures on Hilbert cube manifolds, CBMS Regional Conf. Ser. in Math., no. 28, Amer. Math. Soc., Providence, R.I., 1975.

6. D. W. Curtis, Growth hyperspaces of Peano continua, Trans. Amer. Math. Soc. 238 (1978), 271-283.

7. D. W. Curtis and R. M. Schori, Hyperspaces of Peano continua are Hilbert cubes, Fund. Math. 101 (1978), 19-38.

8. __ Hyperspaces which characterize simple homotopy type, General Topology and Appl. 6 (1976), 153-165.

9. Carl Eberhart, Intervals of continua which are Hilbert cubes, Proc. Amer. Math. Soc. 68 (1978), 220-224.

10. Carl Eberhart and Sam B. Nadler, Jr. Hyperspaces of cones and fans, Proc. Amer. Math. Soc. 77 (1979), 279-288.

11._ The dimension of certain hyperspaces, Bull. Acad. Polon. Sci. Sér. Sci. Math. 19 (1971), $1027-1034$.

12. J. B. Fugate, G. R. Gordh, Jr. and Lewis Lum, Arc-smooth continua, Trans. Amer. Math. Soc. 265 (1981), 545-561.

13. John L. Kelley, Hyperspaces of a continuum, Trans. Amer. Math. Soc. 52 (1942), 22-36.

14. Ronald J. Knill, Cones, products, and fixed points, Fund, Math. 60 (1967), 35-46.

15. R. J. Koch and I. S. Krule, Weak cutpoint ordering on hereditarily unicoherent continua, Proc. Amer. Math. Soc. 11 (1960), 679-681.

16. J. Krasinkeiwicz, Curves which are continuous images of tree-like continua are movable, Fund. Math. 89 (1975), 233-260.

17. K. Kuratowski, Topology, Vol. II, Academic Press, New York, 1968.

18. Stefan Mazurkeiwicz, Sur l'hyperspace d'un continu, Fund. Math. 18 (1932), 171-177.

19. E. E. Moise, Grille decomposition and convexification theorems for compact locally connected continua, Bull. Amer. Math. Soc. 55 (1949), 1111-1121.

20. Sam B. Nadler, Jr., A characterization of locally connected continua by hyperspace retractions, Proc. Amer. Math. Soc. 67 (1977), 167-176.

21. , Hyperspaces of sets, Pure and Appl. Math., vol.49, Dekker, New York, 1978.

22. __ Some basic connectivity properties of Whitney map inverses in $C(X)$, Studies in Topology, Proc. Charlotte Topology Conf., 1974 (Nick M. Stavrakas and Keith R. Allen, Eds.), Academic Press, New York, 1975, pp. 393-410.

23. W Whitney-reversible properties, Fund. Math. 109 (1980), 235-248.

24. Ann Petrus, Contractibility of Whitney continua in $C(X)$, General Topology and Appl. 9 (1978), 275-288. 
25. H. Torunczyk, On CE-images of the Hilbert cube and characterization of Q-manifolds, Fund. Math. 106 (1980), 31-40.

26. L. E. Ward, Jr., Extending Whitney maps, preprint.

27. James E. West, The subcontinua of a dendron form a Hilbert cube factor, Proc. Amer. Math. Soc. 36 (1972), 603-608.

28. Hassler Whitney, Regular families of curves. I, Proc. Nat. Acad. Sci. U.S.A. 18 (1932), 275-278.

29. Gordon Thomas Whyburn, Analytic topology, Amer. Math. Soc. Colloq. Publ., vol. 28, Amer. Math. Soc., Providence, R.I., 1942.

30. M. Wojdyslawski, Sur la contractilité des hyperspaces des continus localement connexes, Fund. Math. 30 (1938), 247-252.

Department of Mathematics, West Virginia University, Morgantown, West Virginia 26506 